Monatsschr Kinderheilkd 2019 167 (Suppl 1):S1-S21

https://doi.org/10.1007/s00112-018-0448-8

Online publiziert: 6. März 2018

(c) Der/die Autor(en) 2018. Dieser Artikel ist

eine Open-Access-Publikation.

\section{Redaktion}

A. Borkhardt, Düsseldorf

S. Wirth, Wuppertal

CrossMark
Z. Szépfalusi $\cdot$ F. Horak $\mathrm{Jr}^{2} \cdot$ T. Eiwegger ${ }^{3} \cdot$ E. Horak $^{4} \cdot$ I. Huttegger ${ }^{5} \cdot$ S. Wolf-

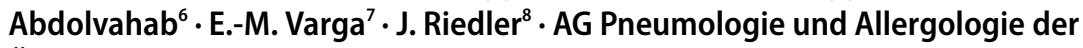
Österreichischen Gesellschaft für Kinder- und Jugendheilkunde

${ }^{1}$ Univ.-Klinik für Kinder und Jugendheilkunde, Medizinische Universität Wien, Wien, Österreich

${ }^{2}$ Allergiezentrum Wien West, Wien, Österreich

${ }^{3}$ Hospital for Sick Children, Toronto, Kanada

${ }^{4}$ Department für Kinder und Jugendheilkunde, Päd. Pneumologie, Medizinische Universität Innsbruck, Innsbruck, Österreich

${ }^{5}$ Univ.-Klinik für Kinder- und Jugendheilkunde, Uniklinikum Salzburg, Salzburg, Österreich

${ }^{6}$ Abteilung für Kinder und Jugendheilkunde, SMZ Ost, Wien, Österreich

${ }^{7}$ Klin. Abt. für Päd. Pulmonologie und Allergologie, Univ.-Klinik für Kinder und Jugendheilkunde, Graz, Österreich

${ }^{8}$ Abteilung für Kinder- und Jugendmedizin, Kardinal Schwarzenbergklinikum, Schwarzach, Österreich

\title{
Allergenspezifische Immuntherapie bei IgE- vermittelten Erkrankungen im Kindes- und Jugendalter
} Übersicht über in Österreich zugelassene
Allergenpräparate - Update 2017

\section{Einleitung}

Warum soll ein nationaler Konsensusreport zur Verwendung der allergenspezifischen Immuntherapie (AIT) im Kindes- und Jugendalter in Österreich erstellt werden?

Neben internationalen Leitlinien [1-5] sowie rezenten Cochrane-Reviews [6-11] liegt eine aktuelle S2-Leitlinie der Deutschen Gesellschaft für Allergologie und klinische Immunologie (DGAKI), des Ärzteverbandes Deutscher Allergologen (ÄDA) und der Gesellschaft für Pädiatrische Allergologie und Umweltmedizin (GPA) 2014 [12] vor. In diesen Veröffentlichungen/Leitlinien wird zwar umfassend auf die medizinischen Aspekte, wie Indikationen, Kontraindikationen

Konsensusreport 2017 der AG Pneumologie und Allergologie der Österreichischen Gesellschaft für Kinder-und Jugendheilkunde.

Z. Szépfalusi ist Leiter der Arbeitsgruppe Pneumologie und Allergologie der Österreichischen Gesellschaft für Kinder und Jugendheilkunde. und Nebenwirkungen, eingegangen, die Empfehlungen basieren aber vorwiegend auf wissenschaftlichen Studiendaten aus dem Erwachsenenalter, obwohl die Bedeutung des frühzeitigen Einsatzes der allergenspezifischen Immuntherapie im Verlauf der Krankheitsentwicklung, d.h. oft bereits im frühesten Kindesalter, betont wird. Neben der zumeist geringen Studienzahl an Kindern und Jugendlichen liegt nunmehr eine gesetzliche Vorgabe der Europäischen Kommission vor, die eine Prüfung der Arzneimittel an Kindern und Jugendlichen vorschreibt, bevor eine Zulassung zur Verwendung an Kindern und Jugendlichen zu erteilen ist (EC No. 1901\&1902/2006, [13]). Übergangsregelungen sind auch festgehalten. Zulassungen werden vonseiten der Pharmaindustrie dementsprechend zunehmend europaweit angestrebt, benötigen aber in vielen Fällen weiterhin eine nationale Bewilligung. Dementsprechend sind nationale Zulassungen von Arzneimitteln und Arzneispezialitäten auch zukünftig für die verschreibenden
Ärzte von Relevanz. Das Angebot an in Österreich registrierten Arzneimitteln und Arzneispezialitäten hat sich in den letzten Jahren stark geändert. Da die Zulassungen für AIT-Arzneimittel und -Spezialitäten bereits das frühe Kleinkindalter (zwischen „ab 2. Lj.“ bis „ab 6. Lj." [Lj: Lebensjahr]) für etliche Produkte umfassen, sind alle Fachärzte (für Kinder- und Jugendheilkunde, Dermatologie, Hals-Nasen-Ohren-Heilkunde, Pneumologie) gefordert und letztendlich verpflichtet, nach gewissenhafter Prüfung der Datenlage aus den zugelassenen Produkten zu wählen. Dieser Anforderung soll in diesem KonsensusreportUpdate 2017 Rechnung getragen werden. Für die Wahl einer AIT soll eine Entscheidungshilfe zur Verfügung gestellt werden, indem alle zurzeit in Österreich erhältlichen Präparate und Eckdaten von Studien aufgelistet werden. Auch diese Liste unterscheidet sich aufgrund der unterschiedlichen Zulassungen in Österreich und Deutschland von jener der oben genannten deutschen Leitlinie 
[12]. Obwohl auf die Indikationen bei der AIT kurz eingegangen wird, möchten wir bezüglich offener Diskussionspunkte und spezieller Aspekte auf die überarbeitete Leitlinie der Arbeitsgemeinschaft der Wissenschaftlichen Medizinischen Fachgesellschaften (AWMF, [12]) hinweisen. Insbesondere kann diese Übersichtsarbeit keine „Reihung“ bestimmter allergenspezifischer Immuntherapeutika in Bezug auf Wirksamkeit und Sicherheitsprofil vornehmen, da hierfür für das Kindes- und Jugendalter kaum Vergleichsstudien vorliegen.

\section{Zielsetzung der Immuntherapie mit Allergenen}

Die AIT (Allergieimpfung, Hyposensibilisierung) setzt als einzige kausale Behandlungsform bei allergischer Rhinokonjunktivitis (RC), allergischem Asthma bronchiale und Insektengiftallergie am Immunsystem an und greift unmittelbar in den Krankheitsprozess ein. Damit werden nicht nur die Allergiesymptome gelindert, sondern es wird auch die zugrunde liegende Ursache der Allergie bekämpft $[9,14,15]$. Die immunologischen Wirkmechanismen werden zunehmend besser verstanden [16-19]. Der allergrößte Teil dieses Wissens wurde aus Untersuchungen an Erwachsenen unter Anwendung der subkutanen spezifischen Immuntherapie („subcutaneous immunotherapy“, SCIT) generiert. Neuere Studien mit dem sublingualen Applikationsweg („sublingual immunotherapy“, SLIT) konnten bekannte Wirkmechanismen der SCIT großteils auch bestätigen. Vor diesem Hintergrund werden die immunologischen Wirkmechanismen wie folgt zusammengefasst [13]:

\section{Immunologische Wirk- mechanismen}

Gemäß dem derzeitigen Stand des Wissens sind die Hauptzielzellen der spezifischen Immuntherapie die T- und die B-Lymphozyten, deren Funktionen selektiv dargestellt werden:

\section{T-Lymphozyten:}

- durch einen „switch“ im T-Lymphozyten-Profil, wobei die dominante Antwort der T-Helfer-Zellen vom Typ 2 (TH2-Antwort mit Überproduktion von Zytokinen wie Interleukin[IL]-4, IL-5, IL-13) zugunsten einer stärkeren TH1Antwort (Interferon- $\gamma[$ IFN- $\gamma]$ ) abgelöst wird [20-23], obwohl dies in manchen Studien nicht nachzuweisen ist [24-26];

- durch Aktivierung regulatorischer T-Lymphozyten, sog. Treg-Zellen, die IL-10 und „transforming growth factor- $\beta$ “ (TGF- $\beta$ ) produzieren und Toleranz vermitteln [20, 27-32] und

- durch Induktion einer verminderten Reaktionsbereitschaft mit abnehmender Zytokinproduktion und verminderter Proliferation nach Stimulation über den T-ZellRezeptor [33-37].

2. B-Lymphozyten:

- Induktion einer gesteigerten allergenspezifischen IgG-Produktion, insbesondere IgG4 [27, 28, 38-41] und

- Reduktion der allergenspezifischen IgE-Produktion (Ig: Immunglobulin; $[16,17,39,42])$.

\section{Allergenextrakte}

Zur SIT werden nichtmodifizierte, native Allergene als wässrige oder physikalisch gebundene (Semidepot-)Extrakte sowie chemisch modifizierte Extrakte (Allergoide, Depigoide) als Semidepotextrakte eingesetzt ([43-45]; - Tab. 1). Allergoide haben weniger reaktive B-Zell-Epitope und eine geringere IgE-Bindung bei Erhalt der T-Zell-Epitop-Bindung und damit der immunogenen Wirkung zur Grundlage; dies soll eine bessere Verträglichkeit bedingen. Vergleichende Untersuchungen von modifizierten Extrakten (Allergoiden/Depigoiden) und nichtmodifizierten Allergenen bei Kindern liegen nicht vor. Bei eingeschränkter Datenlage und Studienqualität ist bei Allergoiden und Depigoiden eine Wirksamkeit und gute Verträglichkeit nachgewiesen worden [46-53].

\section{Wirksamkeit in klinischen Studien}

Bei geeigneter Indikation und richtiger Anwendung sind SCIT und SLIT im Kindesalter als risikoarm anzusehen. Die Rate von systemischen Reaktionen beträgt unter $0,1 \%$ der Injektionen [54, 55]. Die Indikationsstellung zielt primär auf die Verbesserung einer bereits bestehenden Allergiesymptomatik ab. Gerade in der Pädiatrie ergeben sich aufgrund der sequenziellen Dynamik der Allergiesymptome über das Kindesalter durch die AIT aber auch Aspekte im Sinne einer Sekundärprävention allergischer Erkrankungen (z.B. Vorbeugung eines Asthma bronchiale bei bestehender allergischen Rhinitis). Diese Aspekte sind in letzter Zeit neu in die Diskussion geraten, da bisher keine doppelblinde, placebokontrollierte (DBPC-)Studien für diese Altersgruppen vorlagen und damit eine schlüssige Indikationsstellung unter dem Sekundärpräventionsaspekt noch immer weitestgehend aussteht. Dies sollte sich aber nach Beendigung von Studien mit hohen Patientenzahlen und qualitativ hochwertigem Studiendesign bald ändern. Während die Indikation zur AIT bei einer potenziell lebensbedrohlichen Insektengiftallergie prinzipiell altersunabhängig gestellt wird, gilt für Inhalationsallergien, basierend auf den existierenden Richtlinien der World Health Organization (WHO) zur Immuntherapie von 1998 [15], den Allergic Rhinitis and its Impact on Asthma (ARIA) Guidelines von 2001 [56] und den Global Initiative for Asthma (GINA) Guidelines 2017 [57] ein Lebensalter $\geq 5$ Jahre als Empfehlung für den Beginn der Immuntherapie. Die bisher übliche Forderung, wohl aus der Erwachsenenmedizin übernommen, zumindest 2 Beschwerdeperioden mit ähnlicher Allergiesymptomatik vor Erwägung einer AIT aufzuweisen, ist in letzter Zeit der Hoffnung einer Frühintervention im Sinne der Beeinflussung des „allergischen Marsches“ gewichen. Allerdings sind die wenigsten Immuntherapiepräparate vor dem 5. Lebensjahr zugelassen oder lizenziert, und bei noch nicht eindeutiger Symptomatik dient die 2. Episode zur präzisen Indikationsstel- 
lung. Die gängige Therapieform ist eine ganzjährige; für manche Produkte wird eine präsaisonale oder präkosaisonale Applikation empfohlen. Einschlägige pädiatrische Daten liegen hierzu nur eingeschränkt vor $[52,53,55,58]$.

\section{Allergische Rhinokonjunktivitis}

Die allergische RC stellt eine häufige Erkrankung in der Pädiatrie dar. Die AIT bietet (neben der oft eingeschränkten Möglichkeit der Allergenkarenz) den einzigen kausalen Zugang zu dieser Erkrankung. Das „position paper“ der European Academy of Allergy and Clinical Immunology (EAACI) aus dem Jahr 2013 zum Thema allergische Rhinitis im Kindesalter gibt zur AIT folgendes Statement ab: „allergen-specific immunotherapy is helpful in IgE mediated allergic rhinitis and may prevent the progression of allergic disease" [59]. Die Evidenz der Wirksamkeit einer AIT in der Pädiatrie stützt sich auf einige wenige Daten von hoher Qualität und zahlreiche Daten unterschiedlicher Qualität (- Tab. 2 und 3). In diesem Abschnitt wird die Wirksamkeit der SIT bei allergischer RC im Kindesalter anhand rezenter Publikationen beleuchtet.

In der deutschsprachigen Leitlinie zur AIT bei IgE-vermittelter Erkrankung [133] findet sich eine Liste der zur SIT verfügbaren Präparate in Deutschland, die der Therapie-Allergene-Verordnung unterliegen. Diese zulassungsbedürftigen Therapieallergene betreffen Erle, Hasel, Birke, Süßgräser, Hausstaubmilbe, Bienen- und Wespengift. Weitere (seltener indizierte) Therapieallergene sind in dieser Liste nicht angeführt, so ist z. B. eine DBPC-Studie zu Alternaria bei Kindern und Jugendlichen dort nicht zu finden. Die Liste wird halbjährlich aktualisiert (letzter Aktualisierungstand 06/2017); die dort gelisteten Präparate mit den dazugehörigen Studien erfüllen auf den ersten Blick strenge Qualitätskriterien:

- Allergenextrakt und -dosis sind standardisiert,

- randomisiertes, doppelblindes, placebokontrolliertes Studiendesign,

Monatsschr Kinderheilkd 2019 • 167 (Suppl 1):S1-S21

https://doi.org/10.1007/s00112-018-0448-8

@ D Der/die Autor(en) 2018. Dieser Artikel ist eine Open-Access-Publikation.

Z. Szépfalusi · F. Horak Jr · T. Eiwegger · E. Horak · I. Huttegger · S. Wolf-Abdolvahab · E.-M. Varga · J. Riedler · AG Pneumologie und Allergologie der Österreichischen Gesellschaft für Kinder- und Jugendheilkunde

\section{Allergenspezifische Immuntherapie bei IgE-vermittelten Erkrankungen im Kindes- und Jugendalter. Übersicht über in Österreich zugelassene Allergenpräparate - Update 2017}

\section{Zusammenfassung}

Der Konsensusreport 2009 zur allergenspezifischen Immuntherapie (AIT) bei IgEvermittelten (Ig: Immunglobulin) allergischen Erkrankungen im Kindes- und Jugendalter ist aufgrund zunehmend größerer Angebote an Allergenpräparaten und der sehr heterogenen Studienlage entstanden. Mittlerweile erfährt die Landschaft der Immuntherapie eine deutliche Umgestaltung (Verordnung der Europäischen Union [EU], Therapie-AllergeneVerordnung etc.). Geblieben ist die ethische Verpflichtung, Kinder und Jugendliche möglichst mit Allergenpräparaten zu behandeln, die auch in Studien an Kindern und Jugendlichen geprüft sowie als wirksam und sicher eingestuft worden sind (EU-Regulative, European Medicines Agency [EMA]). Die 2017 aktualisierte, für Österreich geltende Liste der Immuntherapiepräparate mit besonderem
Bezug zu Kindern und Jugendlichen wurde nunmehr angepasst. Eine kritische Betrachtung der Studienlandschaft wurde abermals verfolgt. Einige neu auf den Markt gekommene Immuntherapieprodukte zeigen eine verbesserte Datenlage aus Studien, die ausschließlich an Kindern und Jugendlichen durchgeführt wurden. Daher können diese zugelassenen und lizensierten Produkte zur indikationsgerechten Verwendung bei Kindern und Jugendlichen empfohlen werden.

\section{Schlüsselwörter}

Hypersensitivität · Immunologische Desensibilisierung · Sublinguale Immuntherapie . Subkutane Injektionen · State-of-the-ArtReview

\section{Allergen-specific immunotherapy for IgE-mediated diseases in childhood and adolescence. Overview of approved allergen products in Austria_Update 2017}

\section{Abstract}

The consensus report from 2009 on allergenspecific immunotherapy for immunoglobulin $\mathrm{E}(\mathrm{IgE})$ mediated allergic diseases in childhood and adolescence was issued due to the increasingly large offer of allergen preparations and the heterogeneous state of studies. The field of immunotherapy has now undergone a clear restructuring (Regulations of the European Union [EU], TherapyAllergens Regulations etc.). There remains the ethical obligation to treat children and adolescents with allergen preparations, which were also tested in studies including children and adolescents and were classified as being effective and safe (EU regulative, European Medicines Agency [EMA]). The updated list from 2017 of immunotherapy preparations with particular reference to children and adolescents valid for Austria has now been adapted. A critical appraisal of the study situation has been carried out again. Some immunotherapy products which have recently come onto the market show an improved dataset from studies that were carried out exclusively on children and adolescents. These approved and licensed products can therefore be recommended for indications-compliant administration to children and adolescents.

\section{Keywords}

Hypersensitivity · Immunologic desensitization - Sublingual immunotherapy . Subcutaneous injections - State of-the-art review 


\section{Konsensuspapiere}

Tab. 1 Physikochemische Beschaffenheit von Allergenprodukten

Physikalisch Adjuvans Applikation Produkt

gekoppelt

Neue seit 2009 ge-

nerierte Kinderstu-

Seit 2009 nicht

dien oder Studien

mehr zur Verfü-

an Kindern

\section{Nichtmodifiziert}

Wässrige Extrakte

\begin{tabular}{|c|c|c|c|c|c|}
\hline \\
\hline- & - & S.C. & Alutard wässrig ${ }^{\circledR}$ SQ (Biene/Wespe) & - & - \\
\hline- & - & S.C. & Venomenhal ${ }^{\circledR}$ (Biene/Wespe) & - & - \\
\hline- & - & S.C. & AVANZ $^{\circledR}$ (Pollen, Milbe) & $\mathrm{X}$ & - \\
\hline- & - & s.l. & Oralvac plus ${ }^{\circledR}$ (Pollen, Milbe, Tierepithelien, Schimmelpilze) & - & - \\
\hline- & - & s.l. & Sublivac ${ }^{\circledR}$ (Pollen, Milbe, Tierepithelien, Schimmelpilze) & - & - \\
\hline- & - & s.l. & Staloral $300^{\circledR}$ (Pollen, Milbe, Tierepithelien, Schimmelpilze) & - & - \\
\hline- & - & s.l. & $\begin{array}{l}\text { Pangramin SLIT }{ }^{\circledR} \text { (Pollen, Milben, Tierepithelien, Schimmel- } \\
\text { pilze) }\end{array}$ & - & $\mathrm{X}$ \\
\hline- & - & s.l. & $\begin{array}{l}\text { Novo-Helisen }{ }^{\circledR} \text { oral (Pollen, Milben, Tierepithelien, Schim- } \\
\text { melpilze) }\end{array}$ & - & $\mathrm{x}$ \\
\hline- & - & s.l. & SLITone (Pollen, Milben, Tierepithelien, Schimmelpilze) & - & - \\
\hline- & - & s.l. & Oralair ${ }^{\circledR}$ (Gräserpollen) & - & - \\
\hline- & - & s.l. & Grazax $^{\circledR}$ (Gräserpollen) & - & - \\
\hline \multicolumn{6}{|l|}{ Semidepot } \\
\hline L-Tyrosin & - & S.C. & Tyro-SIT ${ }^{\circledR}$ (Pollen, Schimmelpilze, Milben, Tierepithelien) & - & - \\
\hline \multirow[t]{4}{*}{$\begin{array}{l}\text { Aluminium- } \\
\text { hydroxid }\end{array}$} & - & S.C. & $\begin{array}{l}\text { Depot-HAL }{ }^{\circledR} \text { F.I.T. (Pollen, Milben, Tierepithelien, Schim- } \\
\text { melpilze) }\end{array}$ & - & $\mathrm{x}$ \\
\hline & - & S.C. & Alutard SQ ${ }^{\circledR}$ (Pollen, Milben, Tierepithelien, Biene/Wespe) & - & - \\
\hline & - & s.c. & $\begin{array}{l}\text { Novo-Helisen }{ }^{\circledR} \text { Depot (Pollen, Milben, Tierepithelien, } \\
\text { Schimmelpilze) }\end{array}$ & - & - \\
\hline & - & S.c. & Pangramin ${ }^{\circledR}$ (Pollen, Milben) & - & $x$ \\
\hline \multicolumn{6}{|c|}{ Chemisch modifiziert } \\
\hline \multicolumn{6}{|l|}{ Formaldehyd } \\
\hline- & - & S.C. & Acaroid $^{\circledR}$ (Milben) & - & - \\
\hline- & - & - & Allergovit ${ }^{\circledR}$ (Pollen) & - & - \\
\hline \multicolumn{6}{|l|}{ Glutaraldehyd } \\
\hline L-Tyrosin & - & S.c. & Avcarovac $^{\circledR}$ (Milben) & $\mathrm{X}$ & - \\
\hline L-Tyrosin & $\begin{array}{l}\text { Mono } \\
\text { phospho- } \\
\text { ryllipid-A }\end{array}$ & S.C. & Pollinex Quattro ${ }^{\circledR}$ (Pollen) & - & - \\
\hline- & - & S.C. & Purethal ${ }^{\circledR}$ (Pollen, Milben) & - & - \\
\hline
\end{tabular}

- Angabe eines Symptom-MedikationScores und/oder beider Einzel-

Scores,

- Angabe zur statistischen Auswertung,

- statistisch signifikantes Ergebnis und die Wirksamkeit des Verums betragen mindestens $20 \%$ über Placebo.

Allerdings sind bei dieser Liste einige Ausnahmen möglich, daz. B. abweichend von den aktuellen Anforderungen jene vom Paul-Ehrlich-Institut im Jahr der Zulassung geforderten Kriterien maß- geblich sind. Es wird auch erwähnt, dass einzelne Produkte, die in Studien eine Wirksamkeit zeigten, aber den oben genannten Kriterien nicht entsprechen, zur klinischen Dokumentation dennoch angeführt werden. Bei der Durchsicht der publizierten Listen erkennt man rasch die begrenzte Studienlage im Kindesalter [134]. Nachfolgend eine kurze Zusammenfassung dazu:

Die Wirksamkeit einer Immuntherapie im Kindesalter bei allergischer RC durch Gräserpollen ist durch einige hochqualitative Studien, die die oben genannten Kriterien erfüllen, gut belegt. Bei allen gelisteten Studien handelt es sich um die SLIT. Drei Studien wurden mit der Grazax $^{\circledR}$-Sublingualtablette durchgeführt: an 345 Patienten mit saisonaler allergischer RC im Alter von 5 bis 17 Jahren [126], an 253 Patienten mit saisonal allergischer RC mit/ohne Asthma im Alter von 5 bis 16 Jahren [127] und eine Studie mit Oralair ${ }^{\circledR}$-Sublingualtablette an 278 Patienten mit saisonaler allergischer RC im Alter 5 bis 17 Jahren [132]. $\mathrm{Zu}$ ergänzen ist eine gerade zur Publikation akzeptierte Studie (Grazax- 


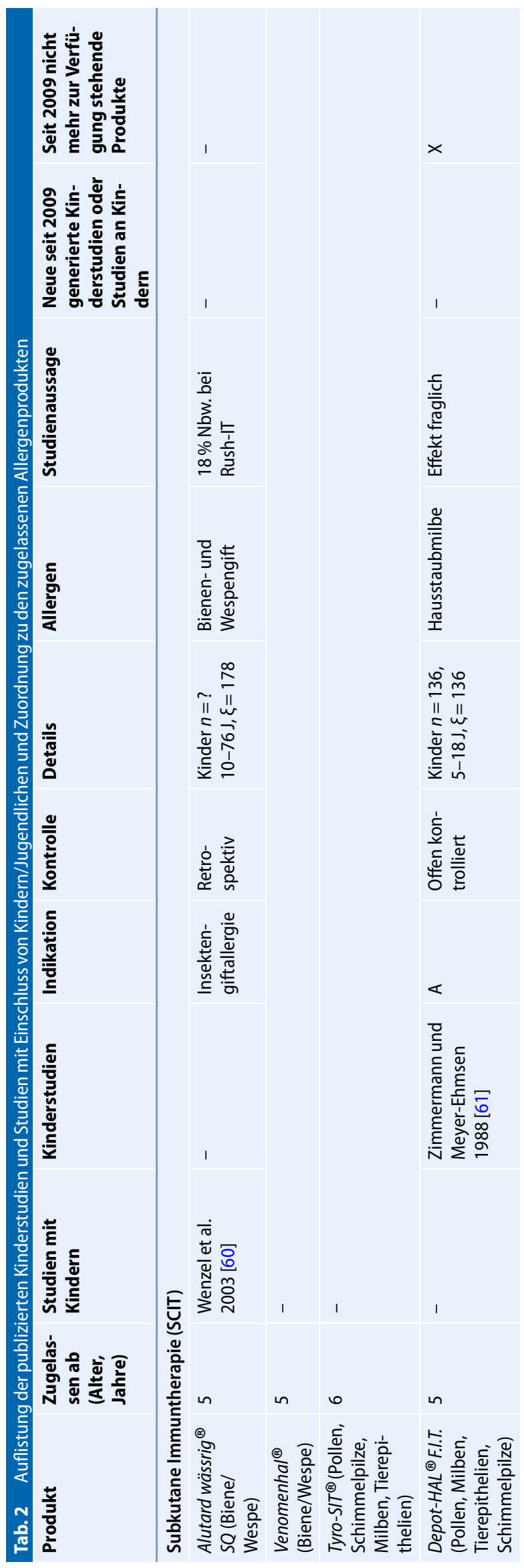

Asthma-Prevention[GAP]-Studie), die an einer Gruppe von 812 Kindern (5 bis 12 Jahre) mit allergischer RC primär den präventiven Effekt einer SLIT mit Grazax $^{\circledR}$ auf die Asthmaentwicklung untersuchte, sekundär aber auch die Wirksamkeit der Behandlung auf RCSymptome und/oder Medikation über 3 Jahre Therapie und 2-Jahre-Follow-up zeigen konnte [135]. Zusammenfassend lässt sich sagen, dass bei der durch eine Gräserpollenallergie ausgelöste allergische RC die Wirksamkeit der SLIT im Kindesalter durch placebokontrollierte Doppelblindstudien mit hohen Patientenzahlen belegt werden konnte. Es fanden sich signifikante Verbesserungen der Symptomen-, Medikamenten- und des kombinierten Symptomen-Medikamenten-Scores sowie eine Verbesserung der Lebensqualität im Vergleich zu Placebo.

Bezüglich einer allergischen RC, ausgelöst durch Baumpollen und Hausstaubmilben, sind entsprechende qualitativ hochstehende Studien im Hinblick auf Wirksamkeit einer Immuntherapie (SCIT und auch SLIT) nur eingeschränkt vorhanden [136-138].

Abgesehen von den Statements der oben genannten Leitlinie gibt es zahlreiche Review-Arbeiten und Metaanalysen zur Effektivität der SIT [139], wobei die inkludierten Studien jedoch nicht oben genannte strenge Qualitätskriterien erfüllen. Diese Publikationen konnten mehrheitlich eine statistisch signifikante Verbesserung der Symptomen- und/oder Medikamenten-Scores aufzeigen, wenngleich Studiendesign, Patientenzahlen, Behandlungsdauer eine große Heterogenität erkennen lassen. Eine Metaanalyse aus dem Jahr 2013 mit 17 doppelblinden randomisierten kontrollierten Studien, eine davon mit Teilnehmenden im Kindesalter, die bei Patienten mit saisonaler allergischer RC mithilfe einer SCIT durchgeführt wurden, zeigte einen positiven Effekt auf Symptome, Medikamentenverbrauch und Lebensqualität [139].

Ebenfalls 2013 wurde eine umfassende Übersicht zur Wirksamkeit der SCIT bei Hausstaubmilbenallergie publiziert, wobei die Studienergebnisse insgesamt heterogen waren und sich in der Arbeit 


\section{Konsensuspapiere}

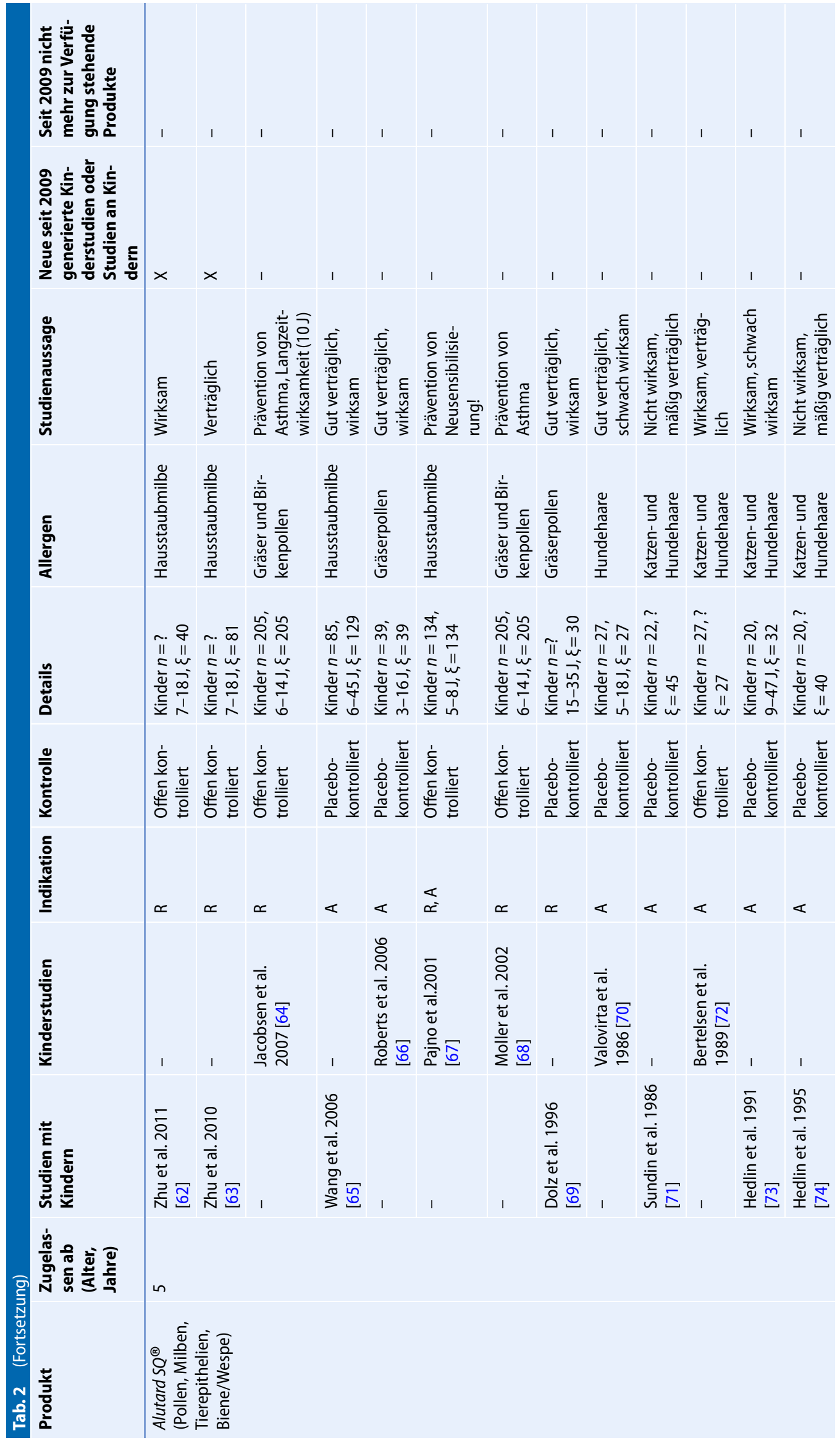




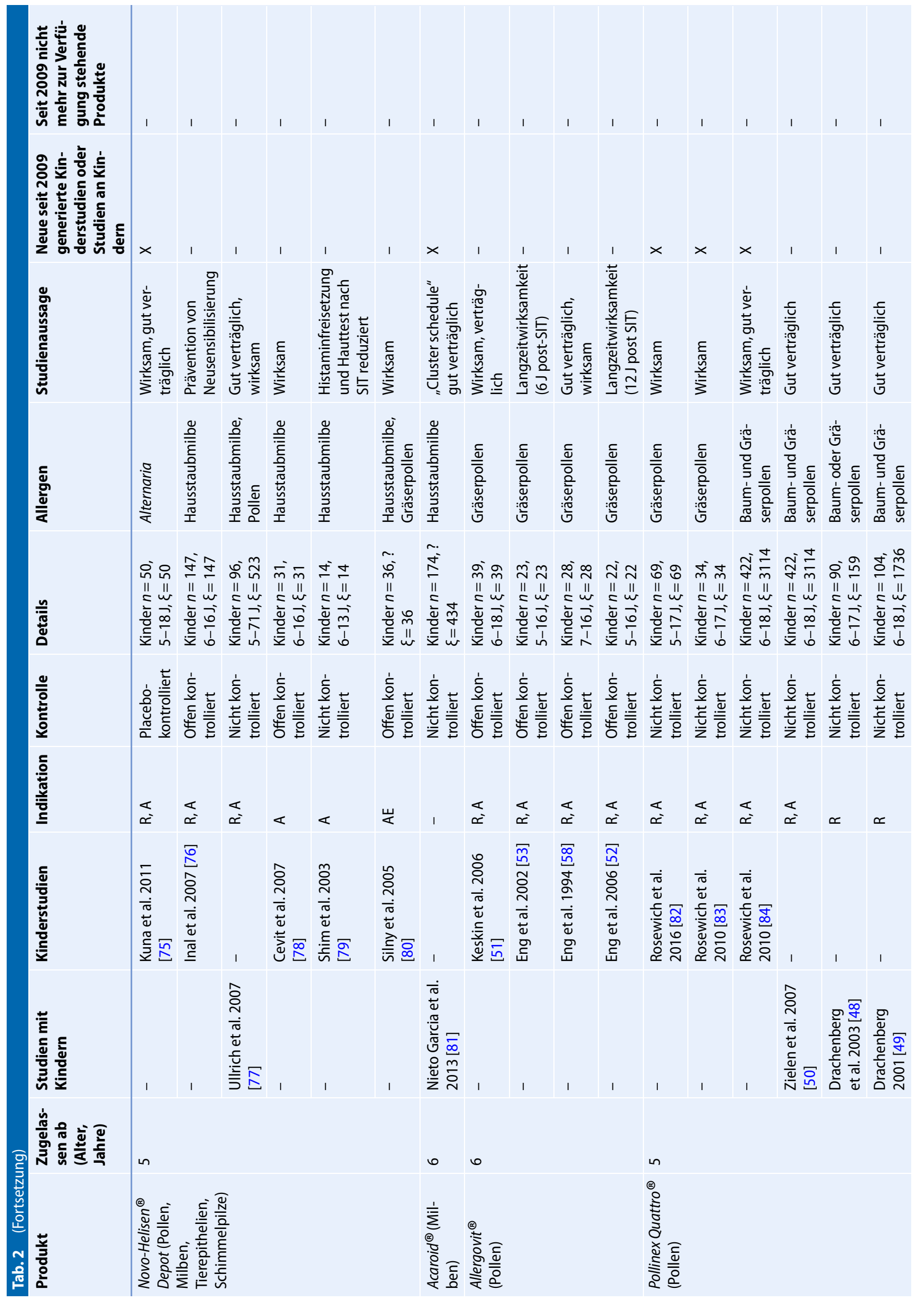




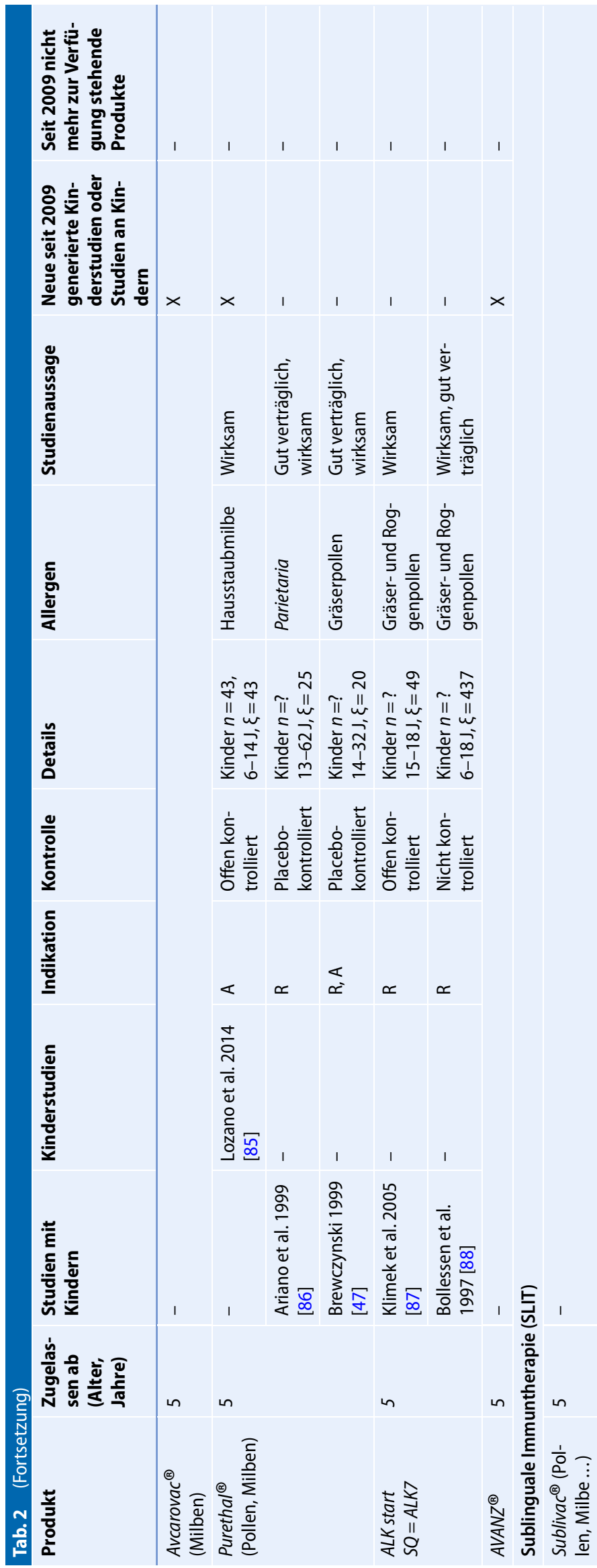

ebenfalls nur eine einzige placebokontrollierte Studie an 30 pädiatrischen $\mathrm{Pa}$ tienten mit allergischer RC und Asthma fand [140].

Ein umfangreicher rezenter pädiatrischer Review zur AIT (SCIT und SLIT) bei allergischer RC bzw. Asthma wurde von Kim et al. publiziert [141]. In diese Arbeit flossen 34 „randomized controlled trials" ein (in Summe 2638 Kinder mit Asthma und/oder allergischer RC): 13 Studien (920 Kinder) SCIT vs. Placebo; 18 Studien (1583 Kinder) SLIT vs. Placebo; drei Studien (135 Kinder) verglichen SCIT mit SLIT. Drei Studien in dieser Arbeit (mit jeweils 205/50/30 Kindern) untersuchten die Effektivität der SCIT bei allergischer RC (Cladosporium, Alternaria bzw. Gräser-/Birkenpollen vs. Placebo) mit der Conclusio einer „moderaten“ Evidenz, was laut Definition der Autoren einer 15\%- bis - $40 \%$ igen Verbesserung im Vergleich zu Placebo entsprach.

Zwölf Studien (insgesamt 1065 Kinder) evaluierten die Effektivität einer SLIT bei allergischer RC, wobei 6 Studien davon an Hausstaubmilbenallergikern durchgeführt wurden. Die Studienergebnisse waren insgesamt sehr heterogen, mit einer breiten Effektivitätsbeurteilung durch die Autoren von „weak“ über "moderate“ bis „strong" [141].

Zusammenfassend lässt sich sagen, dass aufgrund der vorhandenen Datenlage die AIT im Kindesalter bei der allergischen RC eine wirksame Therapieform darstellen kann, wenngleich die Datenfülle zwar umfangreich, aber sehr heterogen und von sehr unterschiedlicher Qualität ist ([7, 47-53, 64, 67-69, 75-77, 86, 94-100, 102-109, 111, $112,114,116,117,122,127,142-145]$; - Tab. 2). Wichtige Aspekte der Langzeitwirkung und des ,disease-modifying effect" bedürfen noch weiterer Untersuchungen.

\section{Allergisches Asthma bronchiale}

Lange Zeit stellte Asthma bronchiale eine Kontraindikation für eine AIT dar. Diese Einschränkung gilt in den letzten Jahren nur mehr für das unkontrollierte Asthma bronchiale. In die aktuellsten Cochrane-Analyse zu Wirksamkeit und Sicherheit der SCIT beim allergischen Asth- 


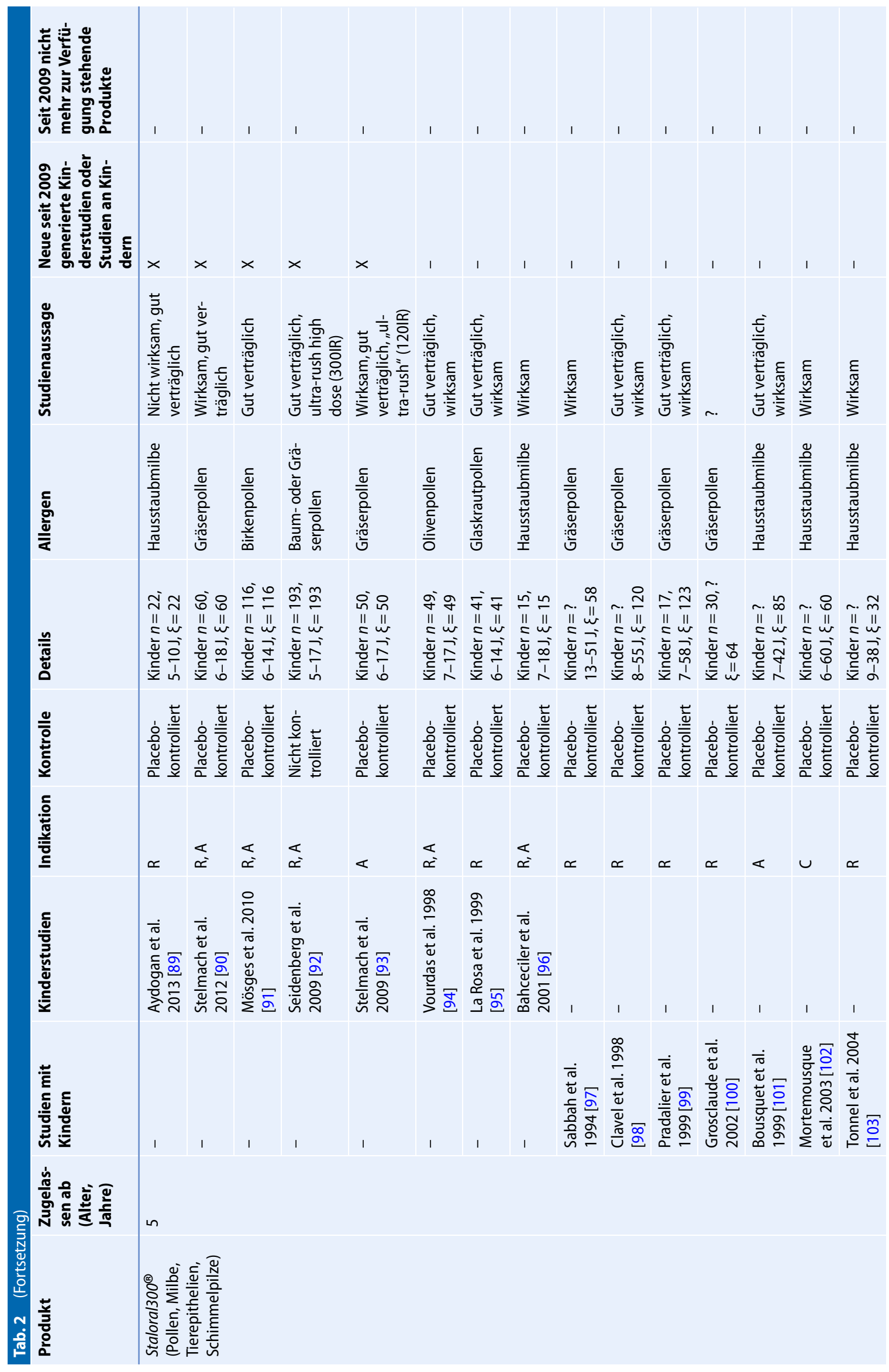




\section{Konsensuspapiere}

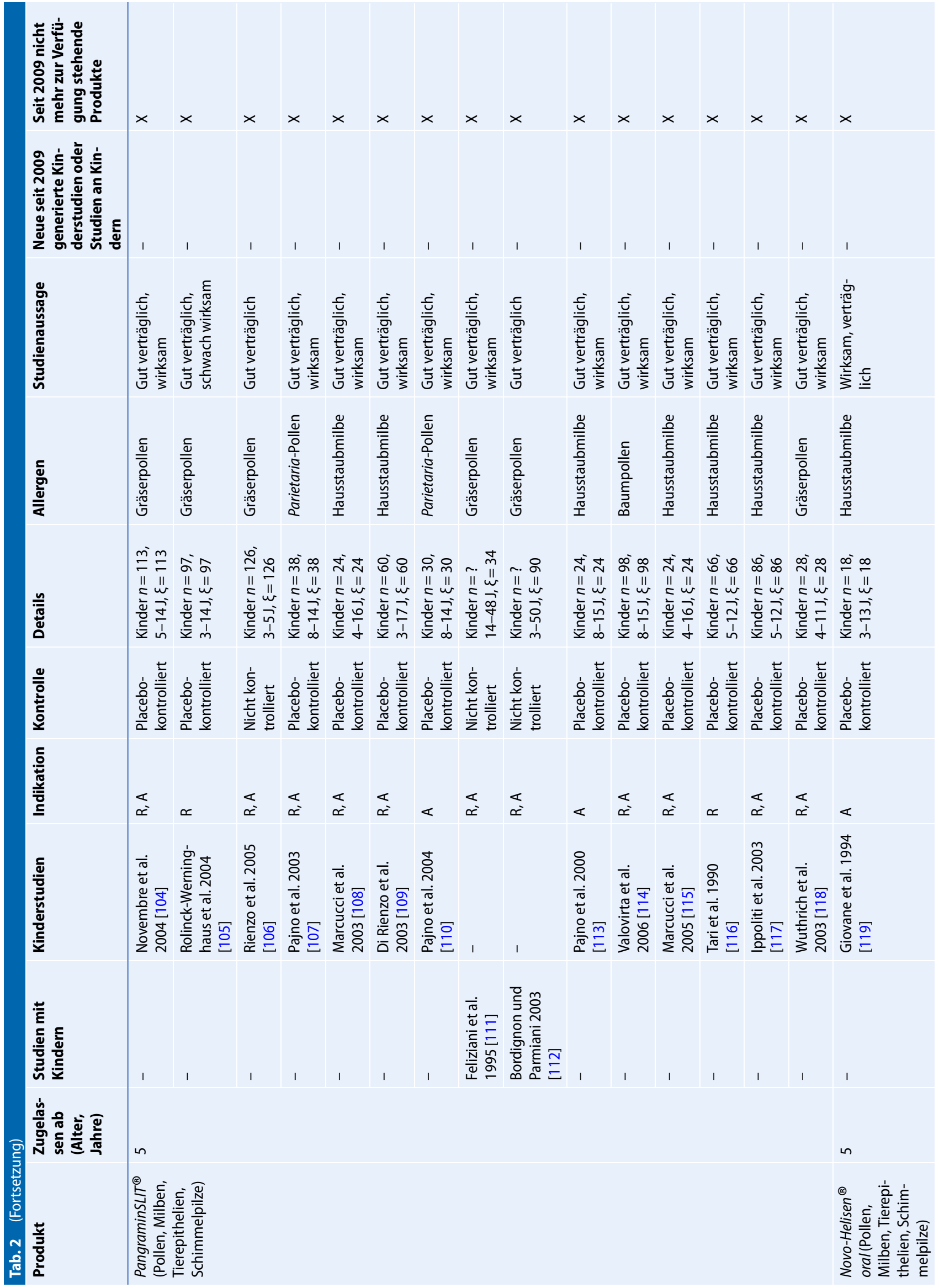




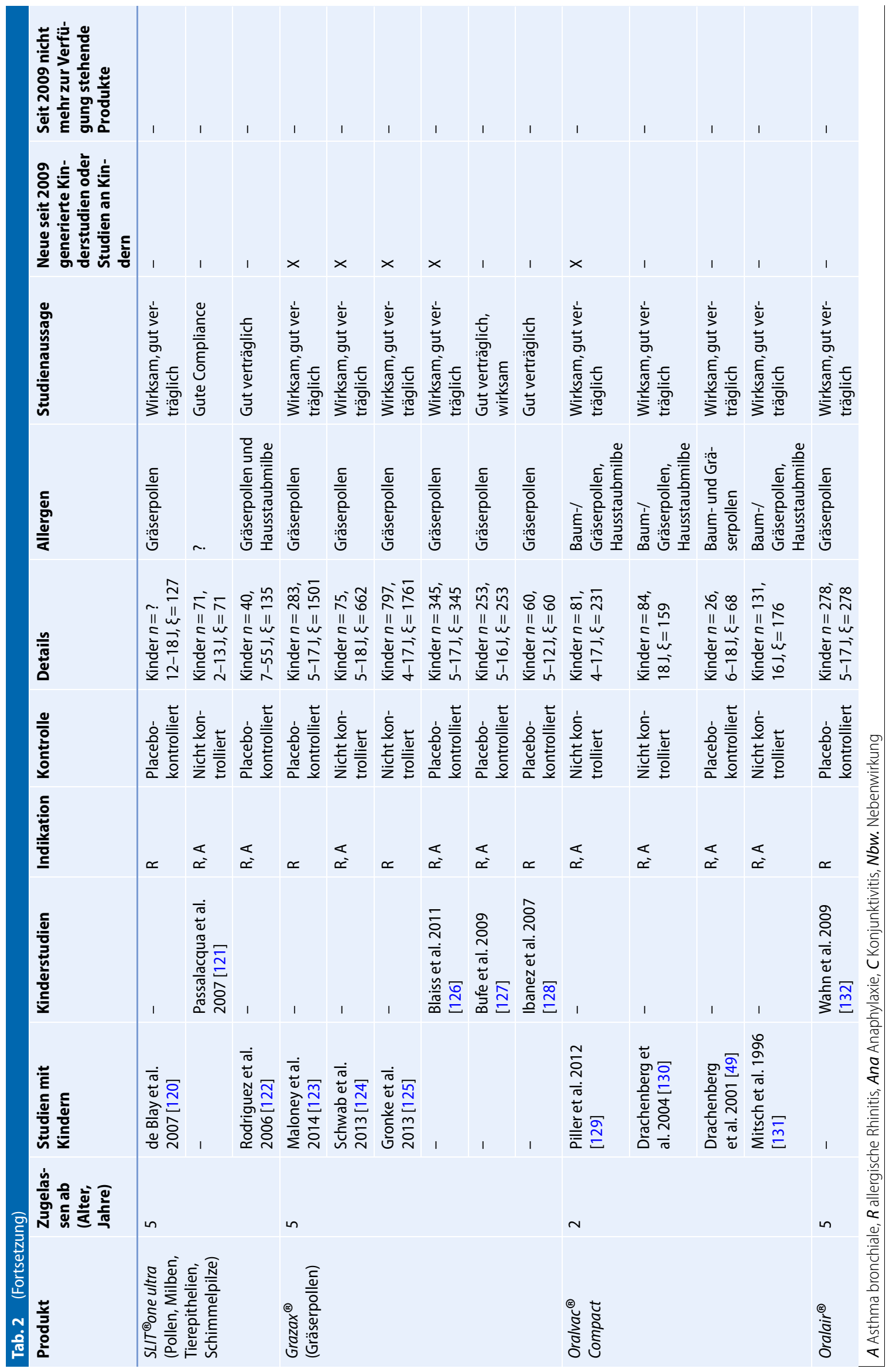


Tab. 3 Auflistung der Allergenprodukte nach Allergenquelle und jeweiligen Kinderstudien bzw. Studien unter Beteiligung von Kindern ${ }^{\mathrm{a}}$ (z. B. 4/5 $=4$ Kinderstudien/5 Studien mit Kinderbeteiligung) Subkutane Immuntherapie (SCIT)

\begin{tabular}{|c|c|c|c|c|}
\hline \multirow{7}{*}{ Gräserpollen } & \multicolumn{2}{|c|}{ Subkutane Immuntherapie (SCIT) } & \multicolumn{2}{|c|}{ Sublinguale Immuntherapie (SLIT) } \\
\hline & Allergovit ${ }^{\circledR}$ & $4 / 0$ & Grazax $^{\circledR}$ & $3 / 3$ \\
\hline & ALK start $\mathrm{SQ}^{\circledR}$ & $0 / 2$ & Oralair ${ }^{\circledR}$ & $1 / 0$ \\
\hline & Alutard SQ ${ }^{\circledR}$ & $3 / 1$ & Oralvac ${ }^{\circledR}$ Compact & $0 / 4$ \\
\hline & $\begin{array}{l}\text { Novo-Helisen }{ }^{\circledR} \\
\text { Depot }\end{array}$ & $1 / 0$ & SLIT $^{\circledR}$ one ultra & $1 / 2$ \\
\hline & Pollinex Quattro ${ }^{\circledR}$ & $3 / 3$ & Staloral $300^{\circledR}$ & $3 / 4$ \\
\hline & Purethal ${ }^{\circledR}$ & $0 / 1$ & & \\
\hline \multirow[t]{2}{*}{ Birkenpollen } & Alutard $\mathrm{SQ}^{\circledR}$ & $2 / 0$ & Oralvac ${ }^{\circledR}$ Compact & $0 / 4$ \\
\hline & Pollinex Quattro ${ }^{\circledR}$ & $1 / 3$ & Staloral $300^{\circledR}$ & $2 / 0$ \\
\hline \multirow[t]{4}{*}{ Hausstaubmilbe } & Acaroid $^{\circledR}$ & $0 / 1$ & $\mathrm{SLIT}^{\circledR}$ one ultra & $1 / 1$ \\
\hline & Alutard SQ ${ }^{\circledR}$ & $1 / 3$ & \multirow[t]{3}{*}{ Staloral $300^{\circledR}$} & \multirow[t]{3}{*}{$2 / 3$} \\
\hline & $\begin{array}{l}\text { Novo-Helisen }{ }^{\circledR} \\
\text { Depot }\end{array}$ & $4 / 1$ & & \\
\hline & Purethal ${ }^{\circledR}$ & $1 / 0$ & & \\
\hline Tierhaare & Alutard $\mathrm{SQ}^{\circledR}$ & $2 / 3$ & \multicolumn{2}{|l|}{ - } \\
\hline Pilzsporen & $\begin{array}{l}\text { Novo-Helisen }{ }^{\circledR} \\
\text { Depot }\end{array}$ & $1 / 0$ & \multicolumn{2}{|l|}{-} \\
\hline Parietaria & Purethal ${ }^{\circledR}$ & $0 / 1$ & Staloral $300^{\circledR}$ & $1 / 0$ \\
\hline Olivenpollen & \multicolumn{2}{|l|}{-} & Staloral $300^{\circledR}$ & $1 / 0$ \\
\hline \multirow[t]{2}{*}{ Insektengifte } & $\begin{array}{l}\text { Alutard wässrig }{ }^{\circledR} \\
\text { SQ }\end{array}$ & $0 / 1$ & \multirow[t]{2}{*}{-} & \\
\hline & Venomenhal ${ }^{\circledR}$ & $1 / 0$ & & \\
\hline
\end{tabular}

ma bronchiale wurden 88 Studien eingeschlossen, 42 davon bei Hausstaubmilben-, 27 bei Pollen-, 10 bei Tierhaarund 2 bei Cladosporium-Allergie [11]. Dabei konnte eine signifikante Verbesserung des Asthma-Symptomen-Scores unter SCIT gezeigt werden. Zudem wurde eine signifikante Reduktion der spezifischen bronchialen Hyperreagibilität nachgewiesen. Die Lungenfunktion wurde durch die SCIT nicht beeinflusst. Bei einem von 9 Patienten kam es durch diese Therapie zu einer systemischen unerwünschten Reaktion (jeglichen Schweregrads). Diese Metaanalyse umfasst Erwachsene und Kinder, und es steht keine, nur auf Kinder bezogene Metaanalyse zur Verfügung [133]. Eine weitere rezente Metaanalyse inkludierte 3 Kinderstudien mithausstaubmilbeninduziertem allergischem Asthma. Die Autoren schlussfolgern, dass die Studien aufgrund geringer Fallzahlen und z.T. ungenügender Kontrolle von Kosensibilisierungen nur beschränkt aussagekräftig sind [140].
Zwei in diesen Metaanalysen nichterfasste Studien konnten bei hausstaubmilbenallergischen Kindern mit Asthma zeigen, dass die SCIT zu einer deutlichen Reduktion der zur Asthmakontrolle notwendigen inhalativen Kortisondosis führt [146], und dass es sowohl für die SCIT als auch für die SLIT zu einer signifikanten Reduktion des totalen AsthmaSymptomen- und Medikamenten-Scores kam [147].

In eine Metaanalyse von 14 Studien zu SLIT und hausstaubmilbeninduziertem allergischem Asthma waren 10 Kinderstudien inkludiert, die sehr heterogene Ergebnisse und große methodische Mängel zeigten [140]. Eine Studie zur SLIT mithilfe einer Gräsertablette bei Kindern mit allergischer RC fand in der Subgruppe der Kinder mit saisonalem Asthma einen positiven Einfluss auf den Asthmaverlauf [127]. Die Autoren der jüngsten Metaanalyse zu SLIT und Hausstaubmilbenasthma bei Kindern zeigten, dass die SLIT die Asthmasymptome reduzieren kann, schlussfolgern aber, dass die Ergebnisse nicht ausreichen, um eine SLIT bei Kindern mit Asthma zu empfehlen [148]. In die größte Studie zu SLIT bei hausstaubmilbenallergischen Patienten waren 604 Jugendliche und Erwachsene über 14 Jahre eingeschlossen. Dabei konnte eine Reduktion der zur Kontrolle des Asthmas notwendigen inhalativen Steroiddosis gezeigt werden [149]. Die jeweiligen Immuntherapiepräparate der unterschiedlichen Hersteller mit angeführter Zulassung/ Lizenzierung, Indikation, Studiendetails $\mathrm{zu}$ vorhandenen Studien mit Kindern oder Kinderstudien sowie den generellen Aussagen der Studien beschreibt • Tab. 2 [7, 47, 50-53, 61, 65-67, 70-79, 82-85, 90, 91, 94, 96, 101, 104, 106-114, 117, $119,122,127,145]$.

\section{Prävention von Asthma und Neusensibilisierungen}

Die Ursprünge aller Studien zur Asthmaprävention mithilfe der AIT sind bereits in den 1960er-Jahren zu finden [150]. Hier wurden Kinder im Alter über 14 Jahre placebokontrolliert, prospektiv verfolgt, und es zeigte sich ein stark protektiver Effekt der SIT (damals noch mit individuellen SIT-Rezepturen).

In der Zwischenzeit konnte in mehreren kontrollierten, allerdings offenen Studien diese Beobachtung teilweise unterstützt werden. Eine aktuelle Metaanalyse, die u.a. die oben genannten Studien berücksichtigt, kommt zu dem Ergebnis, dass zwar ein Kurzzeiteffekt auf die Prävention von Asthma bei bereits bestehender RC $\mathrm{zu}$ erwarten ist („relative risk" [RR] 0,4; $95 \%$-Konfidenzintervall [95\%-KI] 0,30-0,54), dass der Langzeiteffekt derzeit aber noch nicht nachgewiesen werden konnte (RR 0,62; 95\%-KI 0,31-1,23; [10]). Ähnliche Effekte sind bei hausstaubmilbensensibilisierten Kindern in Bezug auf die Verhinderung von Asthma nicht gezeigt worden [10]. In einer aktuellen offenen SLITStudie an 113 gräserallergischen Kindern zeigte sich nach 3 Jahren ein erhöhtes Risiko für die Entstehung von Asthma in der Kontrollgruppe im Vergleich zur SLITGruppe („odds ratio“ [OR] 3,8, $95 \%$ KI 1,5-10,0; [104]). Eine weitere, aktuell zur Publikation akzeptierte Studie unter- 
suchte über 3 Therapie- und 2 Followup-Jahre bei 812 Kindern die Möglichkeit einer Asthmaprävention durch eine sublinguale Gräsertablette (GAP-Studie; $[10,135])$. Der primäre Outcome-Parameter (Zeit bis zum Erstauftreten von Asthma) unterschied sich dabei in der Grazax-Gruppe nicht signifikant von der Placebogruppe ( $8,5 \%$ vs. 9,4\%). Sekundäre Outcome-Parameter zeigten, dass der Anteil der Kinder mit Asthmasymptomen bzw. Asthmamedikamentenbedarf bereits ab dem 2. Therapiejahr in der Aktivgruppe signifikant geringer war als in der Placebogruppe und dieser Effekt auch bis 2 Jahre nach Therapieende anhielt (über die gesamten 5 Jahre: OR 0,67, $p=0,028$ ).

Im Rahmen einer retrospektiven Datenerhebung über Krankenversicherungsunterlagen an Patienten mit einer allergischen RC (Details zu Alter nicht vorliegend), die keine oder eine AIT erhalten hatten, wurde das Auftreten von Asthma bronchiale Jahre danach erfasst. Hierbei zeigte sich eine Prävention von Asthma bronchiale in der Gruppe der AIT-behandelten Patienten [151]. Zusammenfassend sind Kurzzeiteffekte zu beobachten, nicht jedoch Langezeiteffekte [10].

Mehrere Studien weisen darauf hin, dass im Zuge der AIT auch mit einer Reduktion der Neusensibilisierungsraten $\mathrm{zu}$ rechnen ist. Bei Kindern mit einer allergischen RC und/oder einem Asthma bronchiale mit einer Mono- und/oder Oligosensibilisierung zeigen nichtplacebokontrollierte Studien, dass die AIT, als SCIT $[53,67,76,152,153]$ und als SLIT ([109, 154, 155]; Studien z. T. auch retrospektiv), die Häufigkeit von Neusensibilisierungen reduzieren kann (• Tab. 2).

Eine retrospektive Analyse an über 7000 monosensibilisierten Patienten ergab beispielsweise eine wesentlich geringere Rate an Neusensibilisierungen im Vergleich zu einer offenen Kontrollgruppe ( $25 \%$ vs. $75 \%$; [154]). Sehr ähnliche Ergebnisse brachte eine prospektive, offene Studie an 85 Kindern mit einer Hausstaubmilbenallergie und AIT über 5 Jahre [76]. Eine weitere offene, prospektive Studie mit 12 Jahren Follow-up an einer relativ kleinen Gruppe von Kindern unterstützt diese Beobachtung [52, 53].
Kein Unterschied in der Neusensibilisierungsrate, aber ein präventiver Effekt in Bezug auf Asthma konnte in einer weiteren offenen Studie an 60 Kindern, die über 4 bis 5 Jahre eine SLIT erhielten, gezeigt werden [109].

In 2 rezenten Studien mit placebokontrolliertem Design wurde eine präventive SLIT-Therapie gegen Hausstaubmilben bei milbensensibilisierten Kindern ohne allergische Symptome eingesetzt. In beiden Studien wurde die Verhinderung neuer Sensibilisierungen als Zielparameter untersucht. Die präventive SLIT war sicher (i. e. wurde gut vertragen) und lieferte Hinweise auf eine Beeinflussung der immunologischen Parameter, ohne jedoch die Anzahl von Neusensibilisierungen maßgeblich beeinflussen zu können $[40,41]$. In einer weiteren placebokontrollierten Studie an 18 bis 30 Monate alten Kindern mit milder Neurodermitis ohne IgE-Sensibilisierung wurde ein Gemisch an Milben- (Der p1 und Der f1), Katzen- (Fel d1) und Gräserallergen (Phl p5) verabreicht. Hierbei zeigte sich an einem eingeschränkten Patientenkollektiv, dass nach 3 bis 6 Monaten beide Gruppen eine IgE-Sensibilisierung entwickelten; daher wurde die Studie wegen Sicherheitsbedenken beendet [156].

Somit ist für die Fragestellung der Verhinderung von Neusensibilisierungen durch eine AIT bei sensibilisierten, aber noch nicht allergischen Kindern zurzeit die Evidenzlage zu unsicher und daher eine Empfehlung nicht auszusprechen [10]. Bei bereits allergischen Kindern mit einer allergischen RC und/ oder Asthma zeigt oben genannte Metaanalyse eine gewisse Evidenz für einen Kurzzeiteffekt für Neusensibilisierungen (RR 0,33; 95\%-KI 0,12-0,93), jedoch derzeit keine Langzeiteffekte (RR 0,47; $95 \%$-KI 0,08-2,77; [10]). In diesem Sinne argumentiert auch die Allergen Immunotherapy Guideline 2017 der EAACI [157].

\section{Spezifische Immuntherapie mit Insektengiftallergenen}

Zwischen 0,8 und 5\% der Bevölkerung sind von systemischen Überempfindlichkeitsreaktionen nach einem Hymenopterenstich (Biene, Wespe) betroffen
[158-161]. Obwohl Kinder häufiger gestochen werden als Erwachsene, treten systemisch-allergische Reaktionen bei lediglich 0,2-0,8\% der Kinder auf [161]. Die SCIT ist bei Hymenopterengiftallergien eine hocheffektive Behandlungsform und zeigt im Vergleich zur SIT bei Inhalationsallergien eine bessere Wirksamkeit, von $77-84 \%$ bei Bienengiftund von 91-96\% bei Wespengiftallergikern [162-166]. Bei Kindern wurde auch nach Beendigung der SIT ein lang andauernder Schutz nachgewiesen, der mit einer "Relapse“-Rate von $5 \%$ bis zu 20 Jahre nach der ersten systemischallergischen Reaktion, deutlich unter jener von Erwachsenen mit Insektengiftallergie, liegt (10-15\%). Auch eine Rush- sowie Ultra-Rush-Therapie unter stationären Bedingungen ist bei Kindern dokumentiert und bei Bedarf zu empfehlen (in den Sommermonaten mit Stichwiederholungsrisiko; [167, 168]). Die wiederholte Bienenstichexposition als Maß zur Evaluierung der Notwendigkeit einer SIT wird kontrovers diskutiert [169] und in der Praxis sehr selektiv, zumeist in Ermangelung der Insekten und einschlägiger Expertise, nicht oder sehr selten angewandt ([60, 170]; - Tab. 2).

\section{Spezifische Immuntherapie bei atopischem Ekzem}

Da spezifische Sensibilisierungen bei vielen Patienten mit atopischem Ekzem nachweisbar sind, liegt es nahe, Wirksamkeit und Nebenwirkungsprofil der AIT auch bei atopischem Ekzem zu prüfen. Es wurden sowohl randomisierte, placebokontrollierte [67, 171-177] als auch nichtkontrollierte Studien an Kindern [178, 179] mit Hausstaubmilbenallergenextrakten durchgeführt [179]. Weniger Daten liegen zu Gräserpollen- oder Tierallergenextrakten vor. Bei beträchtlicher Heterogenität der Studiendesigns ist ein gewisser Trend zu einer positiven Beeinflussung des atopischen Ekzems erkannt worden (Hausstaubmilbenallergen). Als Maß der Besserung wurden eine Reduktion des Score of Atopic Dermatitis (SCORAD) und des Juckreiz-Scores gefunden. Weitere Studien scheinen vonnöten, um eine klare Empfehlung aussprechen zu können. 
Eine atopische Dermatitis ist derzeit nicht die Indikation für eine SCIT, stellt aber keine Kontraindikation dar. $\mathrm{Zu}$ diesem Schluss kommt auch die S2Leitlinie Neurodermitis der AWMF in der Überarbeitung 2008 sowie in der neuesten Rezension 2014 [180, 181].

\section{Indikationen und Kontraindikationen}

Als Therapieindikation sind die klinischen Krankheitsbilder allergische RC, das kontrollierte allergische Asthma bronchiale und die Insektengiftallergie anzusehen. Als Erfolgsprädiktoren gelten neben der Insektengiftallergie, eine Pollenallergie, eine Hausstaubmilbenallergie, ein schmales Allergenspektrum, eine kurze Erkrankungsdauer, eine geringe Beteiligung der unteren Atemwege, ein junges Lebensalter, eine ganzjährige Behandlung sowie eine ausreichende Compliance/Adhärenz. Allerdings gibt es kaum/keine Kinderstudien, die diese Aussage untermauern würden. $\mathrm{Zu}$ SCIT und SLIT im Kindes- und Jugendalter liegen mittlerweile einige wenige systematische Studienanalysen vor [6, 139-141, 182-188]. Trotzdem ist die Datenlage im Kindesalter immer noch als zu gering anzusehen. Die Wirksamkeit ist je nach Präparat und Allergen unterschiedlich zu bewerten. Die Publikation von systematischen Reviews und Metaanalysen in der Cochrane Library ist aufgrund transparenter und stringenter Bewertungskriterien der Veröffentlichung in Fachzeitschriften überlegen. Eine generelle Befürwortung oder Ablehnung lässt sich nicht herausarbeiten.

Aus dem Erwachsenenbereich abgeleitet, liegt eine Indikation zur SIT mit Allergenen vor, wenn ...

1. der Nachweis einer IgE-vermittelten Sensibilisierung (mit Hauttest und/ oder In-vitro-Diagnostik) und ein eindeutiger Zusammenhang mit klinischer Symptomatik vorliegen,

2. standardisierte bzw. qualitativ hochwertige Allergenextrakte zur Verfügung stehen,

3. der Wirksamkeitsnachweis der geplanten SIT für die jeweilige Indikation erbracht worden ist,
4. Allergenkarenz nicht ausreichend möglich bzw. eine unzureichende Beschwerdefreiheit durch Allergenkarenz erzielbar ist,

5. der Patient ein Alter 5 Jahre aufweist.

Punkte 1-3 sind zu fordern; Punkt 4 kann in die Entscheidung einfließen [12, 133].

Kontraindikationen (absolute und relative) zur AIT bei Kindern liegen vor bei ... [12, 133]

- unkontrolliertem Asthma bronchiale (i. e. klinische Symptomatik und „forced expiratory pressure in 1 second“ $\left.\left[\mathrm{FEV}_{1}\right] 70 \%\right)$,

- Erkrankungen, bei denen Adrenalin kontraindiziert ist,

- schwerwiegenden kardiovaskulären Erkrankungen, Behandlung mit $\beta$-Rezeptoren-Blockern,

- schweren Autoimmunerkrankungen und Immundefizienzen,

- malignen neoplastischen Erkrankungen mit aktuellem Krankheitswert,

- schwerwiegenden systemischen Reaktionen bei durchgeführter AIT in der Vergangenheit,

- unzureichender Compliance.

\section{Durchführung und Applikationsformen}

Grundsätzlich stehen 2 gängige Applikationsformen zur Verfügung:

- subkutan (SCIT),

- sublingual (SLIT; Lösung und selbstauflösende Tablette).

Die Entscheidung für die Verwendung einer AIT in der subkutanen oder sublingualen Applikationsform durch den allergologisch geschulten Arzt erfolgt nach ausführlicher Aufklärung des Patienten/der Eltern, über Dauer, Applikationsart, Wirkung, mögliche Nebenwirkungen und Erfolgsaussichten dieser Therapie unter Berücksichtigung der Behandlungsalternativen. Besonderes Augenmerk ist auf den Zusammenhang zwischen regelmäßiger und ausreichend langer Durchführung der Therapie im Hinblick auf den zu erwartenden Therapieerfolg zu legen. Details zur Durchführung der subkutanen und sublingualen Allergenapplikation sind in einschlä- gigen Literaturstellen nachzulesen [12, 133].

Die schlechte Compliance mangels unzureichender Überprüfbarkeit der Medikamenteneinnahme wird immer wieder als Kritikpunkt zur SLIT angeführt. In einer rezenten pädiatrischen Studie konnte die Compliance jedoch als sehr gut bescheinigt werden [182]. Eine andere Untersuchung fand bei einer sehr großen Patientenzahl einen Abbruch der SLIT über 3 Jahre bei etwa jedem 5. Patienten, womit diese Rate doppelt so hoch war wie bei der SCIT [189]. Es ergeben sich deutliche Unterschiede, ob Daten im Rahmen von klinischen oder in „Real-life-Studien“ erhoben wurden. In Letzteren, untersucht mit Verordnungsdaten der gesetzlichen Krankenversicherung, betragen die gefundenen Persistenzraten zwischen 34 und $57 \%$ im 3. Behandlungsjahr, bezüglich SCIT- und SLIT-Verordnungen [190]. Ein spezielles Augenmerk auf den Aspekt der Compliance bleibt zu fordern, da ansonsten die Therapiesteuerung und damit auch die Therapieerfolge unbefriedigend bleiben. Hilfreich sind fixe Termine für die SCIT sowie eine gute Patientenführung, besonders bei der SLIT mit Recall- und Kontrollterminen.

Die subkutane Applikation, durchzuführen durch den geschulten Arzt, erfolgt nach Prüfung aktueller Infektionen, der Verträglichkeit der letzten Impfung, der Prüfung von Atemwegsobstruktionen, nach eindeutiger Identifizierung der Präparate, streng subkutan in einer angehobenen Hautfalte vorzugsweise eine Handbreit über dem Olekranon an der Streckseite der Oberarme. Nach mindestens 30-minütiger Beobachtung ist die Entlassung des Patienten möglich, eine sportliche Aktivität ist für weitere $2-3 \mathrm{~h}$ verboten. Auch für diese Empfehlung gibt es keine Studiengrundlage, jedoch entspricht sie gelebter Erfahrungspraxis. Entsprechend der Fachinformation wird die Applikation als konventionelles Standardschema, v. a. bei nativen Extrakten in der Steigerungsphase wöchentlich, in der Erhaltungsphase 4-6(-8) wöchentlich durchgeführt [12, 133]. Manche Hersteller verfolgen verkürzte Anfangsschemata, andere Cluster-Therapien, bei Insektengiftallergie eine Rush- oder Ul- 
tra-Rush-Aufdosierung. Die Angaben der Hersteller sind zu beachten. Obwohl keine kontrollierten Studien für die parallele Immuntherapie mit 2 Extrakten in gleicher Sitzung existieren, wird in der klinischen Praxis teilweise zwischen 2 Injektionen (an beiden Oberarmen) ein Abstand von $15 \mathrm{~min}$ eingehalten. Eine Mischung mancher Extrakte ist nicht zulässig, generell ist diese eher nicht zu empfehlen. Die getrennte Applikation von 2 100\%-Extrakten ist auch einer vorgefertigten Mischung (50\% Allergen 1, $50 \%$ Allergen 2) vorzuziehen, da damit eine entsprechende kumulative Dosis für jedes applizierte Allergen gewährleistet ist. Die Gesamtdauer der Immuntherapie beträgt bei Inhalationsallergenen üblicherweise 3 Jahre, bei der Insektenallergie (3) bis 5 Jahre [133]. Bezüglich des Einsatzes einer Prämedikation gibt es kontroverse Ansichten [191, 192]. Bei unerwünschten Begleitreaktionen, laut manchen Autoren auch generell [193], ist eine Prämedikation mit einem Antihistaminikum sinnvoll [194-196].

\section{Sicherheit, Risikofaktoren und unerwünschte Wirkungen}

Schwere, lebensbedrohliche systemische Reaktionen treten sehr selten auf [54, 197-199]. Gemäß den Daten aus dem Paul-Ehrlich-Institut (1991-2000) wurde eine Inzidenz an systematischen Reaktionen von $0,002-0,0076 \%$ pro Injektion bei nichtmodifizierten Allergenextrakten und von $0,0005-0,01 \%$ pro Injektion bei chemisch modifizierten Allergenextrakten (Allergoiden) errechnet [200]. Bei Beachtung von Risikofaktoren sind schwere Reaktionen teilweise absehbar und durch Umsicht und Prophylaxe vermeidbar. Entwickeln sich systemische Nebenwirkungen, reichen sie von einer akuten Bronchialobstruktion bis zum, wenn auch seltenen, anaphylaktischen Schock. Laut einer Untersuchung der American Academy of Allergy, Asthma and Immunology (AAAAI) wurden bei etwa $0,1 \%$ aller durchschnittlich 6,3 Mio. Injektionsvisiten/Jahr systemische Reaktionen beobachtet; fatale Reaktionen traten nicht auf [201, 202].

Bei großen Lokalreaktionen (über $10 \mathrm{~cm}$ Durchmesser) an der Injektions- stelle empfiehlt es sich, bei der nächsten Injektion die Dosis zu wiederholen oder auf eine bereits tolerierte Dosis zurückzugehen, bevor sie erneut gesteigert wird. Große, verzögert nach 6-12 h auftretende Lokalreaktionen erhöhen das individuelle Risiko für systemische Reaktionen nicht. Nach systemischen Reaktionen wird je nach Stärke der Reaktion empfohlen, mindestens 2 bis 3 Stufen oder auf ein Viertel oder ein Achtel der Dosis zurückzugehen oder die Therapie zu beenden. Auch hierzu sind die Angaben der Hersteller zu beachten. Mögliche Einflussfaktoren (z.B. Allergenexposition, Infektionen, unter einer Woche zurückliegende Impfungen, Stress oder eine Mastozytoseerkrankung) sind $\mathrm{zu}$ erfragen, bevor die Dosis erneut bis zur empfohlenen Erhaltungsdosis bzw. individuell verträglichen Höchstdosis gesteigert wird. Kosaisonal ist eine Dosisreduktion zu erwägen, zumindest und besonders im ersten Jahr der AIT. Bei der hochdosierten SLIT in Tablettenform ist die erste Einnahme unter ärztlicher Kontrolle vorzunehmen. Bei einem Präparat wird mit der Erhaltungsdosis begonnen, beim anderen erfolgt eine Aufdosierung über 3 Tage.

\section{Notfalltherapie}

Systemische Reaktionen nach SIT treten in der Mehrzahl der Fälle innerhalb der ersten 30 min auf. Daher müssen Patienten mindestens $0,5 \mathrm{~h}$ nach der Injektion unter Beobachtung bleiben. Das Prinzip der Notfalltherapie ist das schnelle Erkennen einer systemischen Reaktion, gefolgt von raschem therapeutischem Handeln. Adrenalingabe (bevorzugt i.m.; [203-205]), Legen eines i.v.-Zugangs mit Volumengabe, Verabreichung von Steroiden (zur Vorbeugung der Asthmaspätreaktion) und Antihistaminika (Wirksamkeit bei Schweregrad I und II nachgewiesen; [206]) sowie kurz wirksame inhalative Beta-2-Sympathomimetika stellen die wesentlichen Notfalltherapeutika dar. Regelmäßige Notfallschulungen des Personals sind mit entsprechender Dokumentation umzusetzen [207].

\section{Zulassung}

Allergenextrakte liegen als Fertigarzneimittel (Arzneispezialität) und als individuelle Rezepturen vor. Arzneispezialitäten sind zulassungspflichtig, womit eine kontrollierte Prüfung der Wirksamkeit und der Sicherheit für das Präparat erbracht werden muss (trifft in Österreich zurzeit nur für Grazax ${ }^{\circledR}$, Oralair $^{\circledR}$ und Acarizax ${ }^{\circledR} ;[136,137] \mathrm{zu}$; letzteres Präparat ist für Kinder und Jugendliche derzeit nicht zugelassen). Für die Registrierung von Individualrezepturen (alle anderen Immuntherapeutika) ist das Herstellungsverfahren $\mathrm{zu}$ dokumentieren. Eine sorgfältige Prüfung und Bewertung der Wirksamkeits- und Sicherheitsdaten aufgrund der publizierten Studien obliegt dem Verschreiber, i.e. den Fachärzten mehrerer Sonderfächer (z.B. Pädiatrie, Dermatologie, Hals-Nasen-Ohren-Heilkunde, Pneumologie). Um diese Prüfung zu erleichtern, ist in - Tab. 2 die Liste aller Studien zu den jeweiligen in Österreich registrierten und zugelassenen Produkten aufgeführt. Es liegt nahe, dass Produkte mit umfassenden Studiendaten $\mathrm{zu}$ den besser untersuchten zu zählen sind, da deren Wirksamkeit und Sicherheit besser dokumentiert sind und daher eine fundierte Grundlage für die Verwendung dieser Präparate vorliegt. Umgekehrt darf aber auch angemerkt werden, dass nichtvorliegende (und bisher großteils nichtgeforderte) Evidenzdaten sind mit einer bewiesenen Unwirksamkeit des entsprechenden Präparats nicht gleichzusetzen.

\section{Resümee}

Die AIT hat in den letzten Jahrzehnten einen großen Stellenwert in der ursächlichen Behandlung der allergischen $\mathrm{RC}$, des allergischen Asthma bronchiale sowie der Bienen- und Wespengiftallergie mit einem nachhaltigen klinischen und immunologischen Wirkeffekt erhalten. Zahlreiche nationale und, noch in Fertigstellung befindliche, europäische Leit- und Richtlinien, WHO-Berichte, Cochrane- und Metaanalysen belegen die Bedeutung dieser Therapieform. Die Vielzahl der unterschiedlichen Allergen- 
gruppen (Insektengifte, Gräserpollen, Baumpollen, Hausstaubmilben, Tierhaare, Schimmelpilze) sowie die diversen auf dem Markt befindlichen Produkte mit unterschiedlicher Applikationsform und Allergengehalt führen jedoch dazu, dass eine Vergleichbarkeit hinsichtlich der klinischen Wirksamkeit und der immunologischen Veränderungen nicht möglich ist. Darüber hinaus sind die Studienergebnisse bei den Krankheitsbildern allergische RC und allergisches Asthma im Erwachsenenalter nicht generell auf das Kindes- und Jugendalter extrapolierbar. Dies führt dazu, dass die Daten an Kindern und Jugendlichen getrennt von jenen an Erwachsenen $\mathrm{zu}$ betrachten und auszuwerten sind. Obwohl die Gesamtdatenlage zur SIT groß ist, ist jene mit ausschließlichem Einschluss von Kindern und Jugendlichen immer noch zu gering. Dies wird ganz besonders deutlich, wenn man die einzelnen Produkte und deren Spezifizierung auf das eine oder andere Allergen heranzieht. Der vorliegende Konsensusreport 2017, in Analogie zu jenem von 2009 [208], hat sich neuerlich zum Ziel gesetzt, die zugänglichen Daten an Kinderstudien bzw. an Studien mit Einschluss von Kindern tabellarisch zu jedem einzelnen Produkt, das in Österreich eine Registrierung oder Zulassung hat, zusammenzustellen.

Im Sinne der Anwendung von sicheren Medikamenten für Kinder und Jugendliche mit allergischer RC und allergischem Asthma sowie Insektengiftallergie ist zu fordern, dass neben der Verträglichkeit die klinische Wirksamkeit jedes einzelnen Produkts auch für Kinder und Jugendliche in randomisierten Studien getestet wird (als EU-Regulation ab 2006 verpflichtend). Da dies in unterschiedlichem Maß bis dato erfolgt ist, teils auch bedingt durch die unterschiedlichen gesetzlichen Erfordernisse, sollten Fachärzte für Kinder- und Jugendheilkunde bei der Wahl des entsprechenden Immuntherapieprodukts jene Präparate vorziehen, die eine gute Verträglichkeit und eine klinische Wirksamkeit an Kindern- und Jugendlichen nachweisen können. Die Erstellung einer entsprechenden Ranglistung ist nicht möglich, da Vergleichsstudien von Produkten unter- schiedlicher Firmen (zurzeit noch) fehlen.

Festzuhalten ist jedenfalls, dass die AIT auch im Kindes- und Jugendalter neben der oft nur schwer umsetzbaren Allergenkarenz die einzige ursächliche therapeutische Maßnahme in der Behandlung der allergischen RC, des allergischen Asthmas und der Insektengiftallergie darstellt. Bezüglich der Prävention eines Asthma bronchiale bei bestehender allergischer RC ist derzeit von einem Kurzzeiteffekt (bis zu 2 Jahre nach Therapieende) auszugehen; ein langfristiger Vorteil (Langzeiteffekt) ist nicht belegt [10]. Für die Prävention von Neusensibilisierungen durch eine AIT kann derzeit keine befürwortende Empfehlung ausgesprochen werden. Vergleicht man die Häufigkeit des Einsatzes der AIT im Kindes- und Jugendalter mit der hohen Prävalenz dieser allergischen Erkrankungen im Kindesalter, ist ein großer Nachholbedarf gegeben. Dieser Konsensusreport soll daher den Fachärzten für Kinder- und Jugendheilkunde bei ihrer Entscheidung zur AIT helfen, aus jenen Produkten mit entsprechender Zulassung im Kindes- und Jugendalter und auf der Basis der publizierten Datenlage eine differenzierte Auswahl treffen zu können. Es bleibt abschließend zu hoffen, dass die Fachärzte für Kinder- und Jugendheilkunde durch diesen Konsensusreport ermutigt werden, diese Therapieform, aufbauend auf ihrem eigenen fundierten allergologischen Wissen, bei entsprechender Indikation erfolgreich anzuwenden.

Wir wollen aber gleichzeitig auch die Immuntherapiefirmen ermuntern, gemeinsam mit den Fürsprechern dieses Konsensusreports aus der AG Pneumologie und Allergologie der Österreichischen Gesellschaft für Kinderund Jugendheilkunde (ÖGKJ) und den Fachärzten für Kinder- und Jugendheilkunde in Österreich an einer Verbesserung der Datenlage zu ihren Produkten für die sichere Applikation an Kindern und Jugendlichen beizutragen.

\section{Korrespondenzadresse}

\section{Z. Szépfalusi}

Univ.-Klinik für Kinder und Jugendheilkunde, Medizinische Universität Wien

Währinger Gürtel 18-20, 1090 Wien, Österreich zsolt.szepfalusi@meduniwien.ac.at

Mitglieder der AG Pneumologie und Allergologie der Österreichischen Gesellschaft für Kinder- und Jugendheilkunde. Z. Szépfalusi, F. Horak Jr, T. Eiwegger, E. Horak, I. Huttegger, S. Wolf-Abdolvahab, E.-M. Varga, J. Riedler

Funding. Open access funding provided by Medical University of Vienna.

\section{Einhaltung ethischer Richtlinien}

Interessenkonflikt. Z. Szépfalusi, F. Horak Jr, T. Eiwegger, E. Horak, I. Huttegger, S. Wolf-Abdolvahab, E.-M. Varga und J. Riedler für die Österreichische Gesellschaft für Kinder- und Jugendheilkunde geben an, dass kein Interessenkonflikt besteht.

Dieser Beitrag beinhaltet keine von den Autoren durchgeführten Studien an Menschen oder Tieren.

Open Access. Dieser Artikel wird unter der Creative Commons Namensnennung 4.0 International Lizenz (http://creativecommons.org/licenses/by/4.0/deed. de) veröffentlicht, welche die Nutzung, Vervielfältigung, Bearbeitung, Verbreitung und Wiedergabe in jeglichem Medium und Format erlaubt, sofern Sie den/die ursprünglichen Autor(en) und die Quelle ordnungsgemäßnennen, einen Linkzur Creative Commons Lizenz beifügen und angeben, ob Änderungen vorgenommen wurden.

\section{Literatur}

1. Coifman RE, Cox LS (2007) 2006 American Academy of Allergy, Asthma Immunology member immunotherapy practice patterns and concerns. JAllergy Clin Immunol 119:1012-1013

2. Madsen $F$ (2007) EAACI 'Standards for practical allergen-specific immunotherapy'. Allergy 62:332

3. Canonica GW, Baena-Cagnani CE, Bousquet J, Bousquet PJ, Lockey RF, Malling HJ et al (2007) Recommendations for standardization of clinical trials with Allergen Specific Immunotherapy for respiratory allergy. A statement of a World Allergy Organization (WAO) taskforce. Allergy 62:317-324

4. Malling HJ (1999) Allergen-specific immunotherapy. Present state and directions for the future. Allergy 54:30-33

5. Bousquet J, Demoly P (1998) Allergens in 1998: from molecular biology to improved patient care. Allergy 53:549-551

6. Wilson DR, Lima MT, Durham SR (2005) Sublingual immunotherapy for allergic rhinitis: systematic review and meta-analysis. Allergy 60:4-12

7. Passalacqua G, Durham SR (2007) Allergic rhinitis and its impact on asthma update: allergen immunotherapy. J Allergy Clin Immunol 119:881-891

8. Calderon MA, Alves $B$, Jacobson $M$, Hurwitz $B$, Sheikh A, Durham S (2007) Allergen injection immunotherapy for seasonal allergic rhinitis. 
Cochrane Database Syst Rev. https://doi.org/10. 1002/14651858.CD001936.pub2

9. Abramson MJ, Puy RM, Weiner JM (2003) Allergen immunotherapy for asthma. Cochrane Database Syst Rev. https://doi.org/10.1002/14651858. CD001186

10. Kristiansen M, Dhami S, Netuveli G, Halken S, Muraro A, Roberts G et al (2017) Allergen immunotherapy for the prevention of allergy: a systematic review and meta-analysis. Pediatr Allergy Immunol 28:18-29

11. Abramson MJ, Puy RM, Weiner JM (2010) Injection allergen immunotherapy for asthma. Cochrane Database Syst Rev. https://doi.org/10.1002/ 14651858.CD001186.pub2

12. Pfaar $\mathrm{OBC}$, Bufe $A$, Buhl $R$, Ebner $C$, Eng $P$, Friedrichs F, Fuchs T, Hamelmann E, Hartwig-Bade D, Hering T, Huttegger I, Jung K, Klimek L, Kopp MV, Merk $H$, Rabe U, Saloga J, Schmid-Grendelmeier P, Schuster A, Schwerk N, Sitter H, Umpfenbach U, Wedi B, Wöhrl S, Worm M, Kleine-Tebbe J (2014) Guideline on allergen-specific immunotherapy in IgE-mediated allergic diseases. Allergo J Int 23:282-319

13. Regulation of (EC) No $1901 / 2006$ the Euopean Parliament and of the Council of December 2006 on medicinal products for paediatric use and amending Regulation (EEC) No 1768/92, Directive 2001/20/EC, Directive 2001/83/EC and Regulation (EC) No 726/2004. http://ec.europa.eu/enterprise/ pharmaceuticals/eudralex/vol1/reg_2006_1901/ reg 2006 1901 en.pdf.Zugegriffen: Juli 2017

14. CompalatiE, Passalacqua G, Bonini M, Canonica GW (2009) The efficacy of sublingual immunotherapy for house dust mites respiratory allergy: results of a GA2LEN meta-analysis. Allergy 64:1570-1579

15. Bousquet J, Lockey R, Malling HJ (1998) Allergen immunotherapy: therapeutic vaccines for allergic diseases. A WHO position paper. J Allergy Clin Immunol 102:558-562

16. Akdis M, Akdis CA (2007) Mechanisms of allergenspecific immunotherapy. J Allergy Clin Immunol 119:780-791

17. Larche M, Akdis CA, Valenta R (2006) Immunological mechanisms of allergen-specific immunotherapy. Nat Rev Immunol 6:761-771

18. Allam JP, Wurtzen PA, Reinartz M, Winter J, Vrtala $\mathrm{S}$, Chen KW et al (2010) Phl p 5 resorption in human oral mucosa leads to dose-dependent and time-dependent allergen binding by oral mucosal Langerhans cells, attenuates their maturation, and enhances their migratory and TGF-beta1 and IL10-producing properties. J Allergy Clin Immunol 126:638-645e1

19. Fujita H, Soyka MB, Akdis M, Akdis CA (2012) Mechanisms of allergen-specific immunotherapy. Clin Transl Allergy 2:2

20. Bellinghausen I, Metz G, Enk AH, Christmann S, Knop J, Saloga J (1997) Insect venom immunotherapy induces interleukin-10 production and a Th2to-Th1 shift, and changes surface marker expression in venom-allergic subjects. Eur J Immunol 27:1131-1139

21. Jutel M, Pichler WJ, Skrbic D, Urwyler A, Dahinden C, Muller UR (1995) Bee venom immunotherapy results in decrease of IL-4 and IL-5 and increase of IFNgamma secretion in specific allergen-stimulated $T$ cell cultures. JImmunol 154:4187-4194

22. Secrist H, Chelen CJ, Wen Y, Marshall JD, Umetsu DT (1993) Allergen immunotherapy decreases interleukin 4 production in CD4+ T cells from allergic individuals. J Exp Med 178:2123-2130

23. Faith $A$, Richards DF, Verhoef $A$, Lamb JR, Lee $\mathrm{TH}$, Hawrylowicz CM (2003) Impaired secretion of interleukin- 4 and interleukin-13 by allergenspecific $T$ cells correlates with defective nuclear expression of NF-AT2 and jun B: relevance to immunotherapy. Clin Exp Allergy 33:1209-1215

24. Till S, Walker S, Dickason R, Huston D, O'Brien F, Lamb J et al (1997) IL-5 production by allergen-stimulated T cells following grass pollen immunotherapy for seasonal allergic rhinitis. Clin Exp Immunol 110:114-121

25. Wachholz PA, Nouri-Aria KT, Wilson DR, Walker SM, Verhoef A, Till SJ et al (2002) Grass pollen immunotherapy for hayfever is associated with increases in local nasal but not peripheral Th1:Th2 cytokine ratios. Immunology 105:56-62

26. Francis JN, Till SJ, Durham SR (2003) Induction of IL$10+C D 4+C D 25+T$ cells by grass pollen immunotherapy. J Allergy Clin Immunol 111:1255-1261

27. Akdis CA, Blesken T, Akdis M, Wuthrich B, Blaser K (1998) Role of interleukin 10 in specific immunotherapy. JClin Invest 102:98-106

28. Jutel M, Akdis M, Budak F, Aebischer-Casaulta C, Wrzyszcz M, Blaser K et al (2003) IL-10 and TGFbeta cooperate in the regulatory $T$ cell response to mucosalallergens in normal immunity and specific immunotherapy. Eur JImmunol 33:1205-1214

29. Nouri-Aria KT, Wachholz PA, Francis JN, Jacobson MR, Walker SM, Wilcock LK et al (2004) Grass pollen immunotherapy induces mucosal and peripheral IL-10 responses and blocking IgG activity. J Immunol 172:3252-3259

30. Savolainen J, Jacobsen L, Valovirta E (2006) Sublingual immunotherapy in children modulates allergen-induced in vitro expression of cytokine mRNA in PBMC. Allergy 61:1184-1190

31. Ling EM, Smith T, Nguyen XD, Pridgeon C, Dallman $M$, Arbery J et al (2004) Relation of CD4+CD25+ regulatory $\mathrm{T}$-cell suppression of allergen-driven T-cell activation to atopic status and expression of allergic disease. Lancet 363:608-615

32. Akdis M, Verhagen J, Taylor A, Karamloo F, Karagiannidis C, Crameri R et al (2004) Immune responses in healthy and allergic individuals are characterized by a fine balance between allergenspecific T regulatory 1 and T helper 2 cells. J Exp Med 199:1567-1575

33. Kinaciyan T, Jahn-Schmid B, Radakovics A, Zwolfe B, Schreiber C, Francis JN et al (2007) Successful sublingual immunotherapy with birch pollen has limited effects on concomitant food allergy to apple and the immune response to the Bet v 1 homolog Mal d 1. J Allergy Clin Immunol 119:937-943

34. Bohle B, Kinaciyan T, Gerstmayr M, Radakovics A, Jahn-Schmid B, Ebner C (2007) Sublingual immunotherapy induces IL-10-producing $T$ regulatory cells, allergen-specific T-cell tolerance, and immune deviation. J Allergy Clin Immunol 120:707-713

35. Evans R, Pence H, Kaplan H, Rocklin RE (1976) The effect of immunotherapy on humoral and cellular responses in ragweed hayfever. JClin Invest 57:1378-1385

36. Marcotte GV, Braun CM, Norman PS, Nicodemus CF, Kagey-Sobotka A, Lichtenstein LM et al (1998) Effects of peptide therapy on ex vivo T-cell responses. J Allergy Clin Immunol 101:506-513

37. Muller U, Akdis CA, Fricker M, Akdis M, Blesken T, Bettens F et al (1998) Successful immunotherapy with T-cell epitope peptides of bee venom phospholipase A2 induces specific T-cell anergy in patients allergic to bee venom. J Allergy Clin Immunol 101:747-754
38. Wachholz PA, Durham SR (2004) Mechanisms of immunotherapy: IgG revisited. Curr Opin Allergy Clin Immunol 4:313-318

39. Gleich GJ, Zimmermann EM, Henderson LL, Yunginger JW (1982) Effect of immunotherapy on immunoglobulin Eand immunoglobulin Gantibodies to ragweed antigens: a six-year prospective study. J Allergy Clin Immunol 70:261-271

40. ZolkipliZ, Roberts G, Cornelius V, Clayton B, Pearson $S$, Michaelis L et al (2015) Randomized controlled trial of primary prevention of atopy using house dust mite allergen oral immunotherapy in early childhood. J Allergy Clin Immunol 136(6):1541. https://doi.org/10.1016/j.jaci.2015.04.045

41. Szepfalusi Z, Bannert C, Ronceray L, Mayer E, Hassler M, Wissmann E et al (2014) Preventive sublingual immunotherapy in preschool children: first evidence for safety and pro-tolerogenic effects. Pediatr Allergy Immunol 25:788-795

42. van Neerven RJ, Arvidsson M, Ipsen H, Sparholt SH, RakS, Wurtzen PA (2004) A double-blind, placebocontrolled birch allergy vaccination study: inhibition of CD23-mediated serum-immunoglobulin E-facilitated allergen presentation. Clin Exp Allergy 34:420-428

43. Löwer J, Becker W-M, Vieths S (2003) Regulatory control and standardization of allergenic extracts. Druck- und Verlagshaus Sperlich. Arb Paul Ehrlich Inst. Bundesamt Sera Impfstoffe, Frankfurt/Main, S 65-115

44. Kleine-Tebbe J, Fuchs T, Klimek L, Kuhr J, Lepp U, Niggemann B et al (2001) Allergen immunotherapy - a position paper of the German society for allergology and clinical immunology. Pneumologie 55:438-444

45. European Directorate for the Quality of Medicines HealthCare (EDQM) CoE. European Directorate for the Quality of Medicines HealthCare (EDQM), Coucil of Europe (2014) Monograph: Allergen products_Producta Allergenica 2014:1063.

46. Ariano R, Spadolini I, Panzani RC (2001) Efficacy of sublingual specific immunotherapy in Cupressaceae allergy using an extract of $\mathrm{Cu}$ pressus arizonica. A double blind study. Allergol Immunopathol 29:238-244

47. Brewczynski PZ, Kroon AM (1999) Wirksamkeit und Verträglichkeit einer Immuntherapie mit modifizierten Gräserpollenallergenen. Allergologie 22:411-420

48. Drachenberg KJ, Heinzkill M, Urban E, Woroniecki SR (2003) Efficacy and tolerability of short-term specific immunotherapy with pollen allergoids adjuvanted by monophosphoryl lipid A (MLP) for children and adolecents. Allergol Immunopathol 31:270-277

49. Drachenberg KJ, Pfeiffer P Urban E (2001) Sublinguale Immuntherapie. Ergebnisse einer randomisierten, doppelblinden, plazebokontrollierten Multicenterstudie mit einem standardisierten Birken- und Gräser-/Roggenpollenextrakt. Allergologie 24:525-534

50. Zielen S, Metz D, Sommer E, Scherf H-P (2007) Kurzzeit-Immuntherapie mit Allergoiden und dem Adjuvans Monophosphoryl-Lipid-A. Allergologie 30:S1-S8

51. Keskin O, Tuncer A, Adalioglu G, Sekerel BE, Sackesen C, Kalayci O (2006) The effects of grass pollen allergoid immunotherapy on clinical and immunological parametersinchildren withallergic rhinitis. Pediatr Allergy Immunol 17:396-407

52. Eng PA, Borer-Reinhold M, Heijnen IA, Gnehm HP (2006) Twelve-yearfollow-up after discontinuation of preseasonal grass pollen immunotherapy in childhood. Allergy 61:198-201 
53. Eng PA, Reinhold M, Gnehm HP (2002) Long-term efficacy of preseasonal grass pollen immunotherapy in children. Allergy 57:306-312

54. Businco L, Zannino L, Cantani A, Corrias A, Fiocchi A, La Rosa M (1995) Systemic reactions to specific immunotherapy in children with respiratory allergy: a prospective study. Pediatr Allergy Immunol 6:44-47

55. Feng B, Wu J, Chen B, Xiang H, Chen R, Li B et al (2017) Efficacy and safety of sublingual immunotherapy for allergic rhinitis in pediatric patients: a meta-analysis of randomized controlled trials. Am J Rhinol Allergy 31:27-35

56. Bousquet J, Van Cauwenberge P, Khaltaev N (2001) Allergic rhinitis and its impact on asthma. J Allergy Clin Immunol 108:147-334

57. Global Initiative for Asthma (2017)

58. Eng P, Gnehm HE, Joller-Jemelka HI (1994) Clinical and immunologenic effects of preseasonal hyposenitization in children with pollinosis. Monatsschr Kinderheilkd 142:616-622

59. Roberts G, Xatzipsalti M, Borrego LM, Custovic A, HalkenS, Hellings PWetal (2013) Paediatric rhinitis: position paper of the European Academy of Allergy and Clinical Immunology. Allergy 68:1102-1116

60. Wenzel J, Meissner-Kraemer M, Bauer R, Bieber T, Gerdsen R (2003) Safety of rush insect venom immunotherapy. The results of a retrospective study in 178 patients. Allergy 58:1176-1179

61. Zimmermann T, Meyer-Ehmsen K (1988) Diagnostik und Therapie der Sensibilisierung gegen Dermatophagoides pteronyssinus und Dermatophagoides farinae bei Kindern. Allergologie 11:503-508

62. Zhu L, Zhu LP, Chen RX, Tao QL, Lu JH, Cheng $L$ (2011) Clinical efficacy of subcutaneous and sublingual immunotherapy in mite-sensitized patients with allergic rhinitis. Zhonghua Er Bi Yan Hou Tou Jing Wai Ke Za Zhi 46:986-991

63. Zhu L, Lu JH, Xie Q, Wu YL, Zhu LP, Cheng $L$ (2010) Compliance and safety evaluation of subcutaneous versus sublingual immunotherapy in mite-sensitized patients with allergic rhinitis. Zhonghua Er Bi Yan Hou Tou Jing Wai Ke Za Zhi 45:444-449

64. Jacobsen L, Niggemann B, Dreborg S, Ferdousi HA, Halken S, Host A et al (2007) Specific immunotherapy has long-term preventive effect of seasonal and perennial asthma: 10-year followup on the PAT study. Allergy 62:943-948

65. Wang H, Lin X, Hao C, Zhang C, Sun B, Zheng J et al (2006) A double-blind, placebo-controlled study of house dust mite immunotherapy in Chinese asthmatic patients. Allergy 61:191-197

66. Roberts G, Hurley C, Turcanu V, Lack G (2006) Grass pollen immunotherapy as an effective therapy for childhood seasonal allergic asthma. J Allergy Clin Immunol 117:263-268

67. Pajno GB, Barberio G, De Luca F, Morabito L, Parmiani S (2001) Prevention of new sensitizations in asthmatic children monosensitized to house dust mite by specific immunotherapy. A six-year follow-up study. Clin Exp Allergy 31:1392-1397

68. Moller C, Dreborg S, Ferdousi HA, Halken S, Host A, Jacobsen L et al (2002) Pollen immunotherapy reduces the development of asthma in children with seasonal rhinoconjunctivitis (the PAT-study). J Allergy Clin Immunol 109:251-256

69. Dolz I, Martinez-Cocera C, Bartolome JM, Cimarra M (1996) A double-blind, placebo-controlled study of immunotherapy with grass-pollen extract Alutard SQ during a 3-year period with initial rush immunotherapy. Allergy 51:489-500
70. Valovirta E, Viander M, Koivikko A, Vanto T, Ingeman L (1986) Immunotherapy in allergy to dog. Immunologic and clinical findings of a double-blind study. Ann Allergy 57:173-179

71. Sundin B, Lilja G, Graff-Lonnevig V, Hedlin G, Heilborn H, Norrlind Ket al (1986) Immunotherapy with partially purified and standardized animal dander extracts. I. Clinical results from a doubleblind study on patients with animal dander asthma. J Allergy Clin Immunol 77:478-487

72. Bertelsen A, Andersen JB, Christensen J, Ingemann L, Kristensen T, Ostergaard PA (1989) Immunotherapy with dog and cat extracts in children. Allergy 44:330-335

73. Hedlin G, Graff-Lonnevig V, Heilborn H, Lilja G, Norrlind K, Pegelow Ket al (1991) Immunotherapy with cat- and dog-dander extracts. V. Effects of 3 years of treatment. J Allergy Clin Immunol 87:955-964

74. Hedlin G, Heilborn H, Lilja G, Norrlind K, Pegelow KO, Schou C et al (1995) Long-term follow-up of patients treated with a three-year course of cat or dog immunotherapy. J Allergy Clin Immunol 96:879-885

75. Kuna P, KaczmarekJ, Kupczyk M (2011) Efficacy and safety of immunotherapy for allergies to Alternaria alternata in children. J Allergy Clin Immunol 127:502-508e1-6

76. Inal A, Altintas DU, Yilmaz M, Karakoc GB, Kendirl SG, Sertdemir Y (2007) Prevention of new sensitizations by specific immunotherapy in children with rhinitis and/or asthma monosensitized to house dust mite. J Investig Allergol Clin Immuno 17:85-91

77. Ullrich D, Thum-Oltimer S, Mussler S, Jaeschke B (2007) Successful specific subcutaneous immunotherapy (SCIT) with non-modified semi-depot pollen and mite preparations. Allergo J 16:193-198

78. Cevit O, Kendirli SG, Yilmaz M, Altintas DU, Karakoc GB (2007) Specific allergen immunotherapy: effect on immunologic markers and clinical parameters in asthmatic children. J Investig Allergol Clin Immunol 17:286-291

79. Shim JY, Kim BS, Cho SH, Min KU, Hong SJ (2003) Allergen-specific conventional immunotherapy decreases immunoglobulin E-mediated basophi histamine releasability. Clin Exp Allergy 33:52-57

80. Silny W, Czarnecka-Operacz M, Silny P (2005) Efficacy of specific immunotherapy in the treatment of children and youngsters suffering from atopic dermatitis. Part I. Evaluation of clinical score. Wiad Lek 58:47-55

81. Nieto García A, Nevot Falcó S, Carrillo Díaz T, Cumplido Bonny J, Izquierdo Calderón J, Hernández-Peña J (2013) Safety of cluster specific immunotherapy with a modified high-dose house dust mite extract. Eur Ann Allergy Clin Immunol 45:78-83

82. Rosewich M, Girod K, Zielen S, Schubert R, Schulze $J$ (2016) Induction of bronchial tolerance after 1 cycle of monophosphoryl-A-adjuvanted specific immunotherapy in children with grass pollen allergies. Allergy Asthma Immunol Res 8:257-263

83. Rosewich M, Schulze J, Eickmeier O, Telles T, Rose MA, Schubert $R$ et al (2010) Tolerance induction after specific immunotherapy with pollen allergoids adjuvanted by monophosphory lipid A in children. Clin Exp Immunol 160:403-410

84. Rosewich M, Schulze J, Fischer von WeikersthalDrachenberg KJ, Zielen S (2010) Ultra-short course immunotherapy in children and adolescents during a 3-yrs post-marketing surveillance study Pediatr Allergy Immunol 21:e185-e189
85. Lozano J, Cruz MJ, Piquer M, Giner MT, Plaza AM (2014) Assessing the efficacy of immunotherapy with a glutaraldehyde-modified house dust mite extract in children by monitoring changes in clinical parameters and inflammatory markers in exhaled breath. Int Arch Allergy Immunol 165:140-147

86. Ariano R, Kroon AM, Augeri G, Canonica GW, Passalacqua G (1999) Long-term treatment with allergoid immunotherapy with Parietaria. Clinical and immunologic effects in a randomized, controlled trial. Allergy 54:313-319

87. Klimek L, Mewes T, Wolf H, Hansen I, Schnitker J, Mann WJ (2005) The effects of short-term immunotherapy using molecular standardized grass and rye allergens compared with symptomatic drug treatment on rhinoconjunctivitis symptoms, skin sensitivity, and specific nasal reactivity. Otolaryngol Head Neck Surg 133:538-543

88. Bollessen M, Hronung B, Wolf H (1997) Shortterm imunotherapy with molecular standardized grass pollen allergens: Experience from broad application in allergological practice. Allergo $\mathrm{J}$ 6:187-192

89. Aydogan M, Eifan AO, Keles S, Akkoc T, Nursoy MA, Bahceciler NN et al (2013) Sublingual immunotherapy in children with allergic rhinoconjunctivitis mono-sensitized to house-dust-mites: a doubleblind-placebo-controlled randomised trial. Respir Med 107:1322-1329

90. Stelmach I, Kaluzinska-Parzyszek I, Jerzynska J, Stelmach P, Stelmach W, Majak P (2012) Comparative effect of pre-coseasonal and continuous grass sublingual immunotherapy in children. Allergy 67:312-320

91. Mösges R, Graute V, Christ H, Sieber HJ, Wahn U, Niggemann B (2010) Safety of ultra-rush titration of sublingual immunotherapy in asthmatic children with tree-pollen allergy. Pediatr Allergy Immunol 21:1135-1138

92. Seidenberg J, Pajno GB, Bauer CP, La Grutta S, Sieber J (2009) Safety and tolerability of seasonal ultra-rush, high-dose sublingual-swallow immunotherapy in allergic rhinitis to grass and tree pollens: an observational study in 193 children and adolescents. J Investig Allergol Clin Immunol 19:125-131

93. Stelmach I, Kaczmarek-Wozniak J, Majak P, Olszowiec-Chlebna M, Jerzynska J (2009) Efficacy and safety of high-doses sublingual immunotherapy in ultra-rush scheme in children allergic to grass pollen. Clin Exp Allergy 39:401-408

94. Vourdas D, Syrigou E, Potamianou P, Carat F, Batard T, Andre C et al (1998) Double-blind, placebo-controlled evaluation of sublingual immunotherapy with standardized olive pollen extract in pediatric patients with allergic rhinoconjunctivitis and mild asthma due to olive pollen sensitization. Allergy 53:662-672

95. La Rosa M, Ranno C, Andre C, Carat F, Tosca MA, Canonica GW (1999) Double-blind placebocontrolled evaluation of sublingual-swallow immunotherapy with standardized Parietaria judaica extract in children with allergic rhinoconjunctivitis. JAllergy Clin Immunol 104:425-432

96. Bahceciler NN, Isik U, Barlan IB, Basaran MM (2001) Efficacy of sublingual immunotherapy in children with asthma and rhinitis: a double-blind, placebocontrolled study. Pediatr Pulmonol 32:49-55

97. Sabbah A, Hassoun S, Le Sellin J, Andre C, Sicard H (1994) A double-blind, placebo-controlled trial by the sublingual route of immunotherapy with a standardized grass pollen extract. Allergy 49:309-313 
98. ClavelR, Bousquet J,Andre C (1998)Clinical efficacy of sublingual-swallow immunotherapy: a doubleblind, placebo-controlled trial of a standardized five-grass-pollen extract in rhinitis. Allergy 53:493-498

99. Pradalier A, Basset D, Claudel A, Couturier P, Wessel F, Galvain S et al (1999) Sublingual-swallow immunotherapy (SLIT) with a standardized fivegrass-pollen extract (drops and sublingual tablets) versus placebo in seasonal rhinitis. Allergy 54:819-828

100. Grosclaude M, Bouillot P, Alt R, Leynadier F, Scheinmann P, Rufin P et al (2002) Safety of various dosage regimens during induction of sublingual immunotherapy. A preliminary study. Int Arch Allergy Immunol 129:248-253

101. Bousquet J, Scheinmann P, Guinnepain MT, PerrinFayolle M, Sauvaget J, Tonnel AB et al (1999) Sublingual-swallow immunotherapy (SLIT) in patients with asthma due to house-dust mites: a double-blind, placebo-controlled study. Allergy 54:249-260

102. Mortemousque B, Bertel F, De Casamayor J, Verin P Colin J (2003) House-dust mite sublingual-swallow immunotherapy in perennial conjunctivitis: a double-blind, placebo-controlled study. Clin Exp Allergy 33:464-469

103. Tonnel AB, Scherpereel A, Douay B, Mellin B, Leprince D, Goldstein N et al (2004) Allergic rhinitis due to house dust mites: evaluation of the efficacy of specific sublingual immunotherapy. Allergy 59:491-497

104. Novembre E, Galli E, Landi F, Caffarelli C, Pifferi M, De Marco E et al (2004) Coseasonal sublingual immunotherapy reduces the development of asthma in children with allergic rhinoconjunctivitis. JAllergy Clin Immunol 114:851-857

105. Rolinck-Werninghaus C, Hamelmann E, Keil T, Kulig M, Koetz K, Gerstner B et al (2004) The co-seasonal application of anti-lgE after preseasonal specific immunotherapy decreases ocular and nasal symptom scores and rescue medication use in grass pollen allergic children. Allergy 59:973-979

106. Rienzo VD, Minelli M, Musarra A, Sambugaro R, Pecora S, Canonica WG et al (2005) Post-marketing survey on the safety of sublingual immunotherapy in children below the age of 5 years. Clin ExpAllergy 35:560-564

107. Pajno GB, Vita D, Parmiani S, Caminiti L, La Grutta S, Barberio G (2003) Impact of sublingual immunotherapy on seasonal asthma and skin reactivity in children allergic to Parietaria pollen treated with inhaled fluticasone propionate. Clin Exp Allergy 33:1641-1647

108. Marcucci F, Sensi L, Frati F, Bernardini R, Novembre E, Barbato A et al (2003) Effects on inflammation parameters of a double-blind, placebo controlled one-year course of SLIT in children monosensitized to mites. Allergy 58:657-662

109. Di Rienzo V, Marcucci F, Puccinelli P, Parmiani S, Frati F, Sensi L et al (2003) Long-lasting effect of sublingual immunotherapy in children with asthma due to house dust mite: a 10-year prospective study. Clin Exp Allergy 33:206-210

110. Pajno GB, Passalacqua G, Vita D, Caminiti L, Parmiani S, Barberio G (2004) Sublingual immunotherapy abrogates seasonal bronchial hyperresponsiveness in children with Parietaria-induced respiratory allergy: a randomized controlled trial. Allergy 59:883-887

111. Feliziani V, Lattuada G, Parmiani S, Dall'Aglio PP (1995) Safety and efficacy of sublingual rush immunotherapy with grass allergen extracts.
A double blind study. Allergol Immunopathol 23:224-230

112. Bordignon V, Parmiani S (2003) Variation of the skin end-point in patients treated with sublingual specific immunotherapy. J Investig Allergol Clin Immunol 13:170-176

113. Pajno GB, Morabito L, Barberio G, Parmiani S (2000) Clinical and immunologic effects of long-term sublingual immunotherapy in asthmatic children sensitized to mites: a double-blind, placebocontrolled study. Allergy 55:842-849

114. Valovirta $E$, Jacobsen L, Ljorring C, Koivikko A, Savolainen J (2006) Clinical efficacy and safety of sublingual immunotherapy with tree pollen extract in children. Allergy 61:1177-1183

115. Marcucci F, Sensi L, Di Cara G, Salvatori S, Bernini M, Pecora $S$ et al (2005) Three-year followup of clinical and inflammation parameters in children monosensitized to mites undergoing sublingual immunotherapy. Pediatr Allergy Immunol 16:519-526

116. Tari MG, Mancino M, Monti G (1990) Efficacy of sublingual immunotherapy in patients with rhinitis and asthma due to house dust mite. A double-blind study. Allergol Immunopathol 18:277-284

117. IppolitiF, DeSantis W, Volterrani A, LentiL, Canitano $\mathrm{N}$, Lucarelli $S$ et al (2003) Immunomodulation during sublingual therapy in allergic children. Pediatr Allergy Immunol 14:216-221

118. Wuthrich B, Bucher C, Jorg W, Bircher A, Eng P, Schneider Y et al (2003) Double-blind, placebocontrolled study with sublingual immunotherapy in children with seasonal allergic rhinitis to grass pollen. J Investig Allergol Clin Immunol 13:145-148

119. Giovane AL, Bardare M, Passalacqua $G$, Ruffoni $S$, Scordamaglia A, Ghezzi E et al (1994) A threeyear double-blind placebo-controlled study with specific oral immunotherapy to Dermatophagoides: evidence of safety and efficacy in paediatric patients. Clin Exp Allergy 24:53-59

120. de Blay F, Barnig C, Kanny G, Purohit A, Leynadier F, Tunon de Lara J et al (2007) Sublingual-swallow immunotherapy with standardized 3-grass pollen extract: a double-blind, placebo-controlled study. Annals of Allergy, Asthma and Immunology 99:453-461

121. Passalacqua G, Musarra A, Pecora S, Amoroso S, Antonicelli L, Cadario G et al (2007) Quantitative assessment of the compliance with once-daily sublingual immunotherapy in children (EASY project: evaluation of a novel SLIT formulation during a year). Pediatr Allergy Immunol 18:58-62

122. Rodriguez $F$, Boquete $M$, Ibanez $M D$, de la Torre-Martinez F, Tabar Al (2006) Once daily sublingual immunotherapy without updosing - a new treatment schedule. Int Arch Allergy Immuno 140:321-326

123. Maloney J, Bernstein DI, Nelson $\mathrm{H}$, Creticos $\mathrm{P}$, Hebert J, Noonan Met al (2014) Efficacy and safety of grass sublingual immunotherapy tablet, MK7243: a large randomized controlled trial. Ann Allergy Asthma Immunol 112:146-153 e2

124. Schwab JA, Wolf $H$, Schnitker J, Wustenberg $E$ (2013) Safety and tolerability of an intra-seasona initiation of the SQ-standardised grass allergy immunotherapy tablet: a non-interventional observational study investigating the feasibility during routine administration. Clin Drug Investig 33:719-726

125. Gronke C, Wolf H, Schnitker J, Wüstenberg E (2013) Treatment with the SQ-Standardised Grass Allergy Immunotherapy Tablet is in Children,
Adolescents and Adults in Real Life Application An Observational Study. J Allergy Ther 4:5

126. Blaiss M, Maloney J, Nolte H, Gawchik S, Yao R, Skoner DP (2011) Efficacy and safety of timothy grass allergy immunotherapy tablets in North American children and adolescents. J Allergy Clin Immunol 127(e1-4):64-71,71.e1-4

127. Bufe A, Eberle P, Franke-Beckmann E, Funck J Kimmig M, Klimek L et al (2009) Safety and efficacy in children of an SQ-standardized grass allergen tablet for sublingual immunotherapy. J Allergy Clin Immunol 123(e7):167-173e7

128. Ibanez MD, Kaiser F, Knecht R, Armentia A, Schopfer H, Tholstrup B et al (2007) Safety of specific sublingual immunotherapy with SQ standardized grass allergen tablets in children. Pediatr Allergy Immunol 18:516-522

129. Piller M, Boettcher J, Fischer von WeikersthalDrachenberg KJ (2012) Efficacy and patient acceptance of a compact sublingual immunotherap (SLIT). Allergologie 35:9-15

130. Drachenberg KJ, Urban E, Pröll S, Woroniecki SR (2004) Sublingual specific immunotherapy for adults and children: a post-marketing surveillance study. Allergologia et Immunopathologia 32:76-81

131. Mitsch A, Drachenberg KJ (1996) Positive results in a primary multicentric study, specific immunotherapy administered sublingually. TW Paediatrie 9:628-631

132. Wahn U, Tabar A, Kuna P, Halken S, Montagut A, de Beaumont $O$ et al (2009) Efficacy and safety of 5-grass-pollen sublingual immunotherapy tablets in pediatric allergic rhinoconjunctivitis. J Allergy Clin Immunol 123(e3):160-166e3

133. Pfaar O, Demoly P, Gerth van Wijk R, Bonini S, Bousquet J, Canonica GW et al (2014) Recommendations for the standardization of clinical outcomes used in allergen immunotherapy trials for allergic rhinoconjunctivitis: an EAACI Position Paper. Allergy 69:854-867

134. Pfarr et al. www.dgaki.de/leitlinien/s2k-leitliniesit/sit-produkte-studien-zulassung/

135. Valovirta E, Petersen TH, Piotrowska T, Laursen MK, Andersen JS, Sorensen HF et al (2017) Results from the 5-year SQ grass SLIT-tablet asthma prevention (GAP) trial in children with grass pollen allergy. J Allergy Clin Immunol. https://doi.org/10.1016/j. jaci.2017.06.014

136. Nolte H, Bernstein DI, Nelson HS, Kleine-Tebbe J, Sussman GL, Seitzberg D et al (2016) Efficacy of house dust mite sublingual immunotherapy tablet in North American adolescents and adults in a randomized, placebo-controlled trial. J Allergy Clin Immunol 138:1631-1638

137. Okubo K, Masuyama K, Imai T, Okamiya K, Stage BS, Seitzberg D et al (2017) Efficacy and safety of the SQ house dust mite sublingual immunotherapy tablet in Japanese adults and adolescents with house dust mite-induced allergic rhinitis. J Allergy Clin Immunol 139(e10):1840-1848e10

138. Maloney J, Prenner BM, Bernstein DI, Lu S, Gawchik S, Berman G et al (2016) Safety of house dust mite sublingual immunotherapy standardized quality tablet in children allergic to house dust mites. Ann Allergy Asthma Immunol 116:59-65

139. Dretzke J, Meadows A, Novielli N, Huissoon A, FrySmith A, Meads C (2013) Subcutaneous and sublingual immunotherapy for seasonal allergic rhinitis: a systematic review and indirect comparison. JAllergy Clin Immunol 131:1361-1366

140. Calderon MA, Casale TB, Nelson HS, Demoly $P$ (2013) An evidence-based analysis of house dust mite allergen immunotherapy: a call for more 
rigorous clinical studies. J Allergy Clin Immunol 132:1322-1336

141. Kim JM, Lin SY, Suarez-Cuervo C, Chelladurai $Y$ Ramanathan M, Segal JB et al (2013) Allergenspecific immunotherapy for pediatric asthma and rhinoconjunctivitis: a systematic review. Pediatrics 131:1155-1167

142. Fontana V, Holt LJ, Mainland D (1966) Effectiveness of hyposensitization therapy in ragweed hay-fever in children. JAMA 195:985-992

143. WeisnagelJ(1979) Nouvel agent hyposensibilisant dans le traitement de la rhinite allergique saisonniere (fievre des foins) a l'herbe a poux chez Infant: le MRTA (modified ragweed tyrosine adsorbate). Union Med Can 108:685-690

144. Dreborg S, Agrell B, Foucard T, Kjellman NI, Koivikko A, Nilsson S (1986) A double-blind, multicenter immunotherapy trial in children, using a purified and standardized Cladosporium herbarum preparation. I. Clinical results. Allergy 41:131-140

145. Marcucci F, Sensi L, Di Cara G, Incorvaia C, Frati F (2005) Dose dependence of immunological response to sublingual immunotherapy. Allergy 60:952-956

146. Zielen S, Kardos P, Madonini E (2010) Steroid-sparing effects with allergen-specific immunotherapy in children with asthma: a randomized controlled trial. J Allergy Clin Immunol 126:942-949

147. Karakoc-Aydiner E, Eifan AO, Baris S, Gunay E, Akturk E, Akkoc T et al (2015) Long-term effect of Sublingual and subcutaneous immunotherapy in dust mite-allergic children with asthma/rhinitis: a 3-year prospective randomized controlled trial. J Investig Allergol Clin Immunol 25:334-342

148. Liao W, Hu Q, Shen LL, Hu Y, Tao HF, Li HF et al (2015) Sublingual immunotherapy for asthmatic children sensitized to house dust mite: a meta-analysis. Medicine (Baltimore) 94:e701

149. Mosbech $H$, Deckelmann $R$, de Blay F, Pastorello EA, Trebas-Pietras E, Andres LP et al (2014) Standardized quality (SQ) house dust mite sublingual immunotherapy tablet (ALK) reduces inhaled corticosteroid use while maintaining asthma control: a randomized, double-blind, placebo-controlled trial. J Allergy Clin Immunol 134(e7):568-575e7

150. Johnstone DE, Dutton A (1968) The value of hyposensitization therapy for bronchial asthma in children-a 14-year study. Pediatrics 42:793-802

151. Schmitt J, Schwarz K, Stadler E, Wustenberg EG (2015) Allergy immunotherapy for allergic rhinitis effectively prevents asthma: results from a large retrospective cohort study. J Allergy Clin Immunol 136:1511-1516

152. Des Roches A, Paradis L, Menardo JL, Bouges S, Daures JP, Bousquet J (1997) Immunotherapy with a standardized Dermatophagoides pteronyssinus extract. VI. Specific immunotherapy prevents the onset of new sensitizations in children. J Allergy Clin Immunol 99:450-453

153. Reha CM, Ebru A (2007) Specific immunotherapy is effective in the prevention of new sensitivities. Allergol Immunopathol 35:44-51

154. Purello-D'Ambrosio F, Gangemi S, Merendino RA, Isola S, Puccinelli P, Parmiani S et al (2001) Prevention of new sensitizations in monosensitized subjects submitted to specific immunotherapy or not. A retrospective study. Clin Exp Allergy 31:1295-1302

155. Madonini E, Agostinis F, Barra R, Berra A, Donadio D, Pappacoda A et al (2003) Long-term and preventive effects of sublingual allergen-specific immunotherapy: a retrospective, multicentric study. Int J Immunopathol Pharmacol 16:73-79

156. Holt PG, Sly PD, Sampson HA, Robinson P, Loh $\mathrm{R}$, Lowenstein $\mathrm{H}$ et al (2013) Prophylactic use of sublingual allergen immunotherapy in high-risk children: a pilot study. J Allergy Clin Immunol 132(e1):991-993e1

157. Kristiansen M, Dhami S, Netuveli G, Halken S, Muraro A, Roberts $G$ et al (2017) Allergen immunotherapy for the prevention of allergy: A systematic review and meta-analysis. Pediatr Allergy Immunol 28:18-29

158. Golden DB, Kagey-Sobotka A, Norman PS, Hamilton RG, Lichtenstein LM (2004) Outcomes of allergy to insect stings in children, with and without venom immunotherapy. N Engl J Med 351:668-674

159. Schafer T, Ring J (1997) Epidemiology of allergic diseases. Allergy 52:14-22 (discussion 35-6)

160. Schafer T, Kramer U, Dockery D, Vieluf D, Behrend H, Ring J (1999) What makes a child allergic? Analysis of risk factors for allergic sensitization in preschool children from East and West Germany. Allergy Asthma Proc 20:23-27

161. Bilo MB, Bonifazi F (2009) The natural history and epidemiology of insect venom allergy: clinical implications. Clin Exp Allergy 39:1467-1476

162. Menardo JL, Bousquet J, Ville G, Michel FB (1983) Desensitisation with hymenoptera venom in children. Arch Fr Pediatr 40:543-547

163. Urbanek R, Forster J, Kuhn W, Ziupa J (1985) Discontinuation of bee venom immunotherapy in children and adolescents.J Pediatr 107:367-371

164. Valentine MD, Schuberth KC, Kagey-Sobotka A, Graft DF, Kwiterovich KA, Szklo M et al (1990) The value of immunotherapy with venom in children with allergy to insect stings. $\mathrm{N}$ Engl J Med 323:1601-1603

165. Muller U, Helbling A, Berchtold E (1992) Immunotherapy with honeybee venom and yellow jacket venom is different regarding efficacy and safety. J Allergy Clin Immunol 89:529-535

166. Rueff F, Vos B, Oude Elberink J, Bender A, Chatelain R, Dugas-Breit $S$ et al (2014) Predictors of clinical effectiveness of Hymenoptera venom immunotherapy. Clin Exp Allergy 44:736-746

167. Steiss JO, Jodicke B, Lindemann H(2006) A modified ultrarush insect venom immunotherapy protocol for children. Allergy Asthma Proc 27:148-150

168. Laurent J, Smiejan JM, Bloch-Morot E, Herman D (1997) Safety of hymenoptera venom rush immunotherapy. Allergy 52:94-96

169. Schuetze GE, Forster J, Hauk PJ, Friedl K, Kuehr J (2002) Bee-venom allergy in children: long-term predictive value of standardized challenge tests. Pediatr Allergy Immunol 13:18-23

170. Przybilla B, RueffF (1999) Desensitization of allergy to hymenoptera venoms. Wien Med Wochenschr 149:421-428

171. Werfel T, Breuer K, Rueff F, Przybilla B, Worm $M$, Grewe $M$ et al (2006) Usefulness of specific immunotherapy in patients with atopic dermatitis and allergic sensitization to house dust mites: a multi-centre, randomized, dose-response study. Allergy 61:202-205

172. Leroy B, Lachapelle JM, Jacquemin MG, Saint-Remy JM (1993) Immunotherapy of atopic dermatitis by injections of antigen-antibody complexes. Dermatology 186:276-277

173. Ring J (1982) Successful hyposensitization treatment in atopic eczema: results of a trial in monozygotic twins. Br JDermatol 107:597-602

174. Warner JO, Price JF, Soothill JF, Hey EN (1978) Controlled trial of hyposensitisation to Dermato- phagoides pteronyssinus in children with asthma. Lancet 2:912-915

175. Glover MT, Atherton DJ (1992) A double-blind controlled trial of hyposensitization to Dermatophagoides pteronyssinus in children with atopic eczema. Clin Exp Allergy 22:440-446

176. Cadario G, Galluccio AG, Pezza M, Appino A, Milani $M$, Pecora Setal (2007) Sublingual immunotherapy efficacy in patients with atopic dermatitis and house dust mites sensitivity: a prospective pilot study. Curr Med Res Opin 23:2503-2506

177. Bussmann C, Bockenhoff A, Henke $H$, Werfel $T$, Novak N (2006) Does allergen-specific immunotherapy represent a therapeutic option for patients with atopic dermatitis? J Allergy Clin Immunol 118:1292-1298

178. Mastrandrea F, Coradduzza G, Resta F, Cadario G, Rollo MA, Marengo F et al (2005) Increased CD34+ cell peripheral traffic appears to be an unique feature of the allergic inflammation. Eur Ann Allergy Clin Immunol 37:30-33

179. Cox L, Calderon MA (2016) Allergen immunotherapy for atopic dermatitis: is there room for debate? JAllergy Clin Immunol Pract 4:435-444

180. Werfel T, Aberer W, Augustin M, Biedermann T, Fölster-Holst R, Friedrichs F et al (2008) Leitlinien: Neurodermitis. AWMW online.

181. Werfel T, Heratizadeh A, Aberer W, Ahrens F, Augustin M, Biedermann T et al (2016) S2k guideline on diagnosis and treatment of atopic dermatitis - short version. Allergo J Int 25:82-95

182. Roder E, Berger MY, de Groot H, Gerth van Wijk R (2008) Sublingual immunotherapy in youngsters: adherence in a randomized clinical trial. Clin Exp Allergy 38:1659-1667

183. Potter PC (2006) Update on sublingual immunotherapy. Ann Allergy Asthma Immunol 96:22-5

184. Wilson DR, Torres LI, Durham SR (2003) Sublingual immunotherapy for allergic rhinitis. Cochrane Database Syst Rev. https://doi.org/10.1002/ 14651858.CD002893

185. Pajno GB, Caminiti L, Vita D, Barberio G, Salzano G, Lombardo F et al (2007) Sublingual immunotherapy in mite-sensitized children with atopic dermatitis: a randomized, double-blind, placebo-controlled study. J Allergy Clin Immunol 120:164-170

186. Passalacqua G, Guerra L, Pasquali M, Canonica GW (2006) Non-injection routes for allergen immunotherapy: focus on sublingual immunotherapy. Inflamm Allergy Drug Targets 5:43-51

187. Penagos M, Compalati E, Tarantini F, BaenaCagnani R, Huerta J, Passalacqua G et al (2006) Efficacy of sublingual immunotherapy in the treatment of allergic rhinitis in pediatric patients 3 to 18 years of age: a meta-analysis of randomized, placebo-controlled, double-blind trials. Ann Allergy Asthma Immunol 97:141-148

188. Calderon MA, Casale TB, Togias A, Bousquet J, Durham SR, Demoly P (2011) Allergen-specific immunotherapy for respiratory allergies: From meta-analysis to registration and beyond. J Allergy Clin Immunol 127(1):30. https://doi.org/10.1016/j jaci.2010.08.024

189. Pajno GB, Vita D, Caminiti L, Arrigo T, Lombardo F, Incorvaia C et al (2005) Children's compliance with allergen immunotherapy according to administration routes. J Allergy Clin Immunol 116:1380-1381

190. Sieber J, Shah-Hosseini K, Mosges R (2011) Specific immunotherapy for allergic rhinitis to grass and tree pollens in daily medical practice-symptom load with sublingual immunotherapy compared 
to subcutaneous immunotherapy. Ann Med 43:418-424

191. Bonifazi F, Jutel M, Bilo BM, Birnbaum J, Muller U (2005) Prevention and treatment of hymenoptera venom allergy: guidelines for clinical practice. Allergy 60:1459-1470

192. Nielsen L, Johnsen CR, Mosbech H, Poulsen LK, Malling HJ (1996) Antihistamine premedication in specific cluster immunotherapy: a double-blind, placebo-controlled study. J Allergy Clin Immunol 97:1207-1213

193. Jarisch R, Gotz M, Aberer W, Sidl R, Stabel A, Zajc $J$ et al (1988) Reduction of side effects of specific immunotherapy by premedication with antihistaminics and reduction of maximal dosage to 50.000 SQ-U/ml. ArbPaulEhrlich InstBundesamt Sera Impfstoffe, Frankfurt a.M., S 163-175

194. Brockow K, Kiehn M, Riethmuller C, Vieluf D, Berger J, Ring J (1997) Efficacy of antihistamine pretreatment in the prevention of adverse reactions to Hymenoptera immunotherapy: a prospective, randomized, placebo-controlled trial. J Allergy Clin Immunol 100:458-463

195. Reimers A, Hari Y, Muller U (2000) Reduction of side-effects from ultrarush immunotherapy with honeybee venom by pretreatment with fexofenadine: a double-blind, placebo-controlled trial. Allergy 55:484-488

196. Muller UR, Jutel M, Reimers A, Zumkehr J, Huber C, Kriegel C et al (2008) Clinical and immunologic effects of $\mathrm{H} 1$ antihistamine preventive medication during honeybee venom immunotherapy. J Allergy Clin Immunol 122:1001-1007e4

197. Kohli-Wiesner A, Stahlberger L, Bieli C, Stricker T, Lauener R (2012) Induction of specific immunotherapy with hymenoptera venoms using ultrarush regimen in children: safety and tolerance. J Allergy 2012:790910. https://doi.org/10.1155/ 2012/790910

198. Gonzalez FJ, Almirall MC, Herrero AM, De la Torre F, Paris MB (2009) Hymenoptera venom allergy: characteristics, tolerance and efficacy of immunotherapy in the paediatric population. Allergol Immunopathol 37:111-115

199. Malling $\mathrm{HJ}$ (2000) Minimising the risks of allergenspecific injection immunotherapy. Drug Saf 23:323-332

200. Lüderitz-Püchel U, Keller-Stanislawski B, Haustein D (2001) Neubewertung des Risikos von Test- und Therapieallergenen. Eine Analyse der UAW-Meldungen von 1991 bis 2000. Bundesgesundheitsblatt Gesundheitsforschung Gesundheitsschutz 44:709-718

201. Burks AW, Calderon MA, Casale T, Cox L, Demoly P, Jutel $M$ et al (2013) Update on allergy immunotherapy: American Academy of Allergy, Asthma Immunology/European Academy of Allergy and Clinical Immunology/PRACTALL consensus report. JAllergy Clin Immunol 131(e3):1288-1296e3

202. Epstein TG, Liss GM, Murphy-Berendts K, Bernstein DI (2013) AAAAI and ACAAI surveillance study of subcutaneous immunotherapy, Year 3: what practices modify the risk of systemic reactions? Ann Allergy Asthma Immunol 110:274-278, 278.e1

203. SimonsFE(2004)First-aid treatment of anaphylaxis to food: focus on epinephrine. J Allergy Clin Immunol 113:837-844

204. Simons FE, Gu X, Johnston LM, Simons KJ (2000) Can epinephrine inhalations be substituted for epinephrine injection in children at risk for systemic anaphylaxis? Pediatrics 106:1040-1044

205. Sheikh A, Shehata YA, Brown SG, Simons FE (2009) Adrenaline for the treatment of anaphylaxis: cochrane systematic review. Allergy 64:204-212
206. Simons FE (2003) H1-Antihistamines: more relevant than ever in the treatment of allergic disorders. J Allergy Clin Immunol 112:42-52

207. Sampson HA, Munoz-Furlong A, Bock SA, Schmitt C, Bass R, Chowdhury BA et al (2005) Symposium on the definition and management of anaphylaxis: summary report. J Allergy Clin Immunol 115:584-591

208. Szépfalusi Z, Emminger W, Eitelberger F, Gotz M, Grillenberger A, Horak E et al (2009) Allergen-specific Immunotherapy for children and adolescents - a review on available products in Austria. Wien Klin Wochenschr 121:648-660 